\title{
THE CANADA-FRANCE ECLIPTIC PLANE SURVEY-FULL DATA RELEASE: THE ORBITAL STRUCTURE OF THE KUIPER BELT*
}

\author{
J.-M. Petit ${ }^{1,2}$, J. J. Kavelaars ${ }^{3}$, B. J. Gladman ${ }^{2}$, R. L. Jones ${ }^{2,3}$, J. Wm. Parker ${ }^{4}$, C. Van Laerhoven ${ }^{2,5}$, \\ P. Nicholson ${ }^{6}$, G. Mars ${ }^{7}$, P. Rousselot ${ }^{1}$, O. Mousis ${ }^{1}$, B. Marsden ${ }^{8,11}$, A. Bieryla ${ }^{4}$, \\ M. TAYlor ${ }^{9}$, M. L. N. AshbY ${ }^{8}$, P. Benavidez ${ }^{10}$, A. Campo Bagatin ${ }^{10}$, and G. Bernabeu ${ }^{9}$ \\ ${ }^{1}$ Institut UTINAM, CNRS-UMR 6213, Observatoire de Besançon, BP 1615, 25010 Besançon Cedex, France \\ ${ }^{2}$ Department of Physics and Astronomy, University of British Columbia, Vancouver, BC, Canada \\ ${ }^{3}$ Herzberg Institute of Astrophysics, National Research Council of Canada, Victoria, BC V9E 2E7, Canada \\ ${ }^{4}$ Planetary Science Directorate, Southwest Research Institute, Boulder, CO 80302, USA \\ ${ }^{5}$ Department of Planetary Sciences, University of Arizona, Tucson, AZ 85721-0092, USA \\ ${ }^{6}$ Department of Astronomy, Cornell University, Space Sciences Building, Ithaca, NY 14853, USA \\ ${ }^{7}$ Observatoire de la Cote d'Azur, BP 4229, F-06304 Nice Cedex 4, France \\ ${ }^{8}$ Harvard-Smithsonian Center for Astrophysics, Cambridge, MA 02138, USA \\ ${ }^{9}$ Department of Physics and Astronomy, University of Victoria, Victoria, BC V8W 2Y2, Canada \\ ${ }^{10}$ Departamento de Fisica, Ingenieria de Sistemas y Teoria de la Señal, E.P.S.A., Universidad de Alicante, \\ Apartado de Correos 99, Alicante 03080, Spain \\ Received 2011 May 25; accepted 2011 August 1; published 2011 September 15
}

\begin{abstract}
We report the orbital distribution of the trans-Neptunian objects (TNOs) discovered during the Canada-France Ecliptic Plane Survey (CFEPS), whose discovery phase ran from early 2003 until early 2007. The follow-up observations started just after the first discoveries and extended until late 2009. We obtained characterized observations of $321 \mathrm{deg}^{2}$ of sky to depths in the range $g \sim 23.5-24.4 \mathrm{AB}$ mag. We provide a database of 169 TNOs with high-precision dynamical classification and known discovery efficiency. Using this database, we find that the classical belt is a complex region with sub-structures that go beyond the usual splitting of inner (interior to 3:2 mean-motion resonance [MMR]), main (between 3:2 and 2:1 MMR), and outer (exterior to 2:1 MMR). The main classical belt ( $a=40-47 \mathrm{AU}$ ) needs to be modeled with at least three components: the "hot" component with a wide inclination distribution and two "cold" components (stirred and kernel) with much narrower inclination distributions. The hot component must have a significantly shallower absolute magnitude $\left(H_{g}\right)$ distribution than the other two components. With $95 \%$ confidence, there are $8000_{-1600}^{+1800}$ objects in the main belt with $H_{g} \leqslant 8.0$, of which $50 \%$ are from the hot component, $40 \%$ from the stirred component, and 10\% from the kernel; the hot component's fraction drops rapidly with increasing $H_{g}$. Because of this, the apparent population fractions depend on the depth and ecliptic latitude of a trans-Neptunian survey. The stirred and kernel components are limited to only a portion of the main belt, while we find that the hot component is consistent with a smooth extension throughout the inner, main, and outer regions of the classical belt; in fact, the inner and outer belts are consistent with containing only hot-component objects. The $H_{g} \leqslant 8.0$ TNO population estimates are 400 for the inner belt and 10,000 for the outer belt to within a factor of two ( $95 \%$ confidence). We show how the CFEPS Survey Simulator can be used to compare a cosmogonic model for the orbital element distribution to the real Kuiper Belt.
\end{abstract}

Key words: Kuiper Belt: general - surveys

Online-only material: color figures

\section{INTRODUCTION}

The minor body populations of the solar system provide, via their orbital and physical properties, windows into the dynamical and chemical history of the solar system. Recognition of the structural complexity in the trans-Neptunian region has led to models that describe possible dynamical evolutionary paths, such as a smooth migration phase for Neptune (Malhotra 1993), the large-scale re-ordering of the outer solar system (Tsiganis et al. 2005; Thommes et al. 1999), the scattering of now-gone rogue planets (Gladman \& Chan 2006), or the close passage of

\footnotetext{
* Based on observations obtained with MegaPrime/MegaCam, a joint project of the Canada-France-Hawaii Telescope (CFHT) and CEA/DAPNIA, at CFHT which is operated by the National Research Council (NRC) of Canada, the Institute National des Sciences de l'Universe of the Centre National de la Recherche Scientifique (CNRS) of France, and the University of Hawaii. This work is based in part on data products produced at the Canadian Astronomy Data Centre as part of the CFHT Legacy Survey, a collaborative project of NRC and CNRS.

${ }^{11}$ Deceased.
}

a star (Ida et al. 2000). Evaluating these models is fraught with dangers due to observational biases affecting our knowledge of the intrinsic populations of the trans-Neptunian region (see Kavelaars et al. 2008; Jones et al. 2010, for discussion of these issues). Over the past twenty years, many different Kuiper Belt surveys (those with more than 10 detections include Jewitt et al. 1996; Larsen et al. 2001; Trujillo et al. 2001; Gladman et al. 2001; Allen et al. 2002; Millis et al. 2002; Elliot et al. 2005; Petit et al. 2006; Jones et al. 2006; Schwamb et al. 2010) have been slowly building up a sample, albeit with differing flux and pointing biases. Jones et al. (2006) enumerated the aspects of surveys that must be carefully recorded and made public if quantitative comparisons with models are to be made.

The primary goal of the Canada-France Ecliptic Plane Survey (CFEPS) is the production of a catalog of transNeptunian objects (TNOs) combined with a precise account of the observational biases inherent to that catalog. The description of the biases, combined with provisioning of a "survey simulator," enables researchers to quantitatively compare the outcome 
of their model simulations to the observed TNO populations. In Jones et al. (2006) we described our initial "pre-survey" and general motivation for this project, and Kavelaars et al. (2009) (P1 hereafter) described the first year of operation of this survey (the L3 data release). This manuscript describes the observations that make up the integrated seven years of the project and provide our complete catalog (the L7 release) of near-ecliptic detections and characterizations along with fully linked highquality orbits. In summary, the "products" of the CFEPS consist of the following four items:

1. a list of detected CFEPS TNOs, associated with the block of discovery;

2. a characterization of each survey block;

3. a Survey Simulator that takes a proposed Kuiper Belt model, exposes it to the known detection biases of the CFEPS blocks, and produces simulated detections to be compared with the real detections; and

4. the CFEPS-L7 model population.

In Sections 2 and 3, we describe the observation and characterization of the CFEPS TNO sample. The dynamical classification of all tracked TNOs in our sample is given in Section 4. In Section 5, we update our parameterized model of the main and inner classical Kuiper Belt (P1) and give an improved estimate of the total number of objects in each of these dynamical sub-populations. We also extend our model to the non-resonant, non-scattering part of the belt beyond the 2:1 mean-motion resonance (MMR) with Neptune. Section 6 gives an order of magnitude estimate of the scattering disk's population. Section 7 demonstrates the use of our Survey Simulator to compare the results of a cosmogonic model to the CFEPS detections. Finally in Section 8, we present our conclusions and put our findings in perspective.

\section{OBSERVATIONS AND INITIAL REDUCTIONS}

The discovery component of the CFEPS project imaged $\sim 320 \mathrm{deg}^{2}$ of sky, almost all of which was within a few degrees of the ecliptic plane. Discovery observations occurred in blocks of $\approx 16$ fields acquired using the Canada-France-Hawaii Telescope (CFHT) MegaPrime camera which delivered discovery image quality (FWHM) of 0.7-0.9 arcseconds in queue-mode operations. The $0.96 \times 0.94$ MegaPrime FOV is paved by 36 individual 4600x2048 CCDs, each pixel having a scale of $0^{\prime \prime} 187$.

The CFEPS designation of a "block" of discovery fields was a leading "L" followed by the year of observations $(3,4,5$, and 7 ) and then a letter representing the two week period of the year in which the discovery observations were acquired (example: L3f occurred in the second half of 2003 March). Discovery observations occurred between 2003 March and 2005 July plus one block of fields (L7a) observed in 2007 January. The CFEPS presurvey block (Jones et al. 2006) in 2002 also consisted of a single contiguous sky patch. To enhance our sensitivity to the latitude distribution of the Kuiper Belt we also acquired two survey blocks of $11 \mathrm{deg}^{2}$ each, at $\sim 10^{\circ}$ ecliptic latitude (L5r) and $\sim 20^{\circ}$ ecliptic latitude (L5s). Each of the discovery blocks was searched for TNOs using our Moving Object Pipeline (MOP; see Petit et al. 2004). Table 1 provides a summary of the survey fields, imaging circumstances, and detection thresholds, both for CFEPS and for the presurvey. Figure 1 presents the sky coverage of our discovery blocks. For a detailed description of the initial CFEPS observing plan, field sequencing, and follow-up strategy see Jones et al. (2006) and P1.

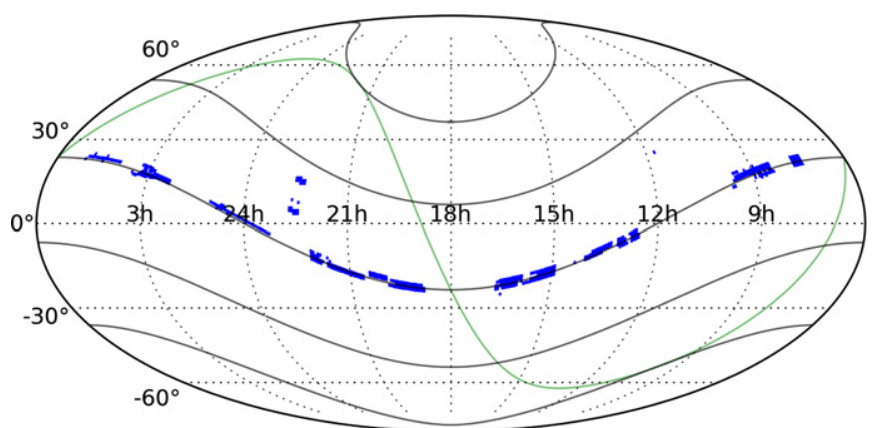

Figure 1. Geometry of the CFEPS discovery blocks. The R.A. and decl. grid is indicated with dotted lines. The black solid curves show constant ecliptic latitudes of $-60^{\circ},-30^{\circ}, 0^{\circ}, 30^{\circ}, 60^{\circ}$ from bottom to top. The remaining curve indicates the plane of the Milky Way.

(A color version of this figure is available in the online journal.)

\section{SAMPLE CHARACTERIZATION}

The photometric calibration of the discovery triplets to a common reference frame and determination of our detection efficiency is required for our survey simulator analysis. It is presented in Appendix B and the photometric measurements of all CFEPS TNOs acquired in photometric conditions are given in Table 7.

We characterized the magnitude-dependent detection probability of each discovery block by inserting artificial sources in the images and running these images through our detection pipeline to recover these artificial sources. We used the $g$ filter at CFHT for all our discovery observations, except for block L3h which was acquired using the $r$ filter. For that block's fields, we shifted the limits to a nominal $g$ value using a color of $(g-r)=0.70$, corresponding to the mean $(g-r)$ color of our full CFEPS sample. The TNOs in each block that have a magnitude brighter than that block's $40 \%$ detection probability are considered to be part of the CFEPS characterized sample. Because detection efficiencies below $\sim 40 \%$ determined by human operators and our MOP diverge-MOP accepts more faint objects, at the expense of false detections-(Petit et al. 2004), and since characterization is critical to the CFEPS goals, we chose not to utilize the sample faint ward of the measured $40 \%$ detection-efficiency level for quantitative science (although we report these discoveries, many of which were tracked to precise orbits). The characterized CFEPS sample consists of 196 objects of the 231 discovered (see Table 7 for a list of these TNOs). The fraction of objects detected brightward of our cutoff is consistent with the shape of the TNO luminosity function (Petit et al. 2008) and typical decay in detection efficiency due to gradually increasing stellar confusion and the rapid falloff at the signal-to-noise ratio limit.

Our discovery and tracking observations were made using short exposures designed to maximize the efficiency of detection and tracking of the TNOs in the field. These observations do not provide the high-precision flux measurements necessary for possible classification based on broadband colors of TNOs and we do not comment here on this aspect of the CFEPS sample.

\section{TRACKING AND LOST OBJECTS}

Tracking during the first opposition was done using the builtin follow-up of the CFEPS project. Subsequent tracking, over the next three oppositions, occurred at a variety of facilities, including CFHT. The observational efforts outside CFHT are summarized in Table 2. In spring 2006 the CFEPS project made 
Table 1

Summary of Field Positions and Detections

\begin{tabular}{|c|c|c|c|c|c|c|c|c|c|c|c|}
\hline \multirow[b]{2}{*}{ Block } & \multirow{2}{*}{$\begin{array}{l}\text { R.A. } \\
\text { (hr) }\end{array}$} & \multirow{2}{*}{$\begin{array}{l}\text { Decl. }^{\text {a }} \\
\text { (deg) }\end{array}$} & \multirow{2}{*}{$\begin{array}{l}\text { Fill }^{\mathrm{b}} \\
\text { Factor }\end{array}$} & \multicolumn{2}{|c|}{ Charact. Det. $^{c}$} & \multirow{2}{*}{$\begin{array}{c}\text { Geometry } \\
(\operatorname{deg} \times \operatorname{deg})\end{array}$} & \multicolumn{2}{|c|}{ Discovery } & \multirow{2}{*}{$\begin{array}{l}\text { Limit }^{\mathrm{d}} \\
g_{A B}\end{array}$} & \multicolumn{2}{|c|}{ Detection Limits ${ }^{\mathrm{e}}$} \\
\hline & & & & Disc. & Track. & & (date) & filter & & rate $(" / \mathrm{hr})$ & direction $(\mathrm{deg})$ \\
\hline L3f & $12: 42$ & $-04: 33$ & 0.80 & 3 & 2 & $4 \times 4$ & 2003 Mar 24 & G.MP9401 & 23.75 & 1.7 to 5.1 & -10.0 to 50.0 \\
\hline L3h & $13: 03$ & $-06: 48$ & 0.81 & 14 & 11 & $4 \times 4$ & 2003 Apr 26 & R.MP9601 & $24.43^{f}$ & 0.8 to 6.2 & 5.6 to 41.6 \\
\hline $\mathrm{L} 3 \mathrm{q}$ & 22:01 & $-12: 04$ & 0.89 & 9 & 7 & $4 \times 4$ & 2003 Aug 31 & G.MP9401 & 24.08 & 1.2 to 6.2 & -38.0 to -2.0 \\
\hline L3s & $19: 43$ & $-01: 20$ & 0.87 & 5 & 5 & $14 \times 1$ & 2003 Sep 23 & G.MP9401 & 23.95 & 0.8 to 8.0 & -42.6 to -5.0 \\
\hline L3w & $04: 33$ & $22: 21$ & 0.87 & 13 & 11 & $16 \times 1$ & 2003 Dec 16 & G.MP9401 & 24.25 & 0.8 to 6.0 & -29.0 to 11.0 \\
\hline L3y & $07: 30$ & $21: 48$ & 0.85 & 10 & 10 & $4 \times 4$ & 2003 Dec 24 & G.MP9401 & 24.08 & 1.7 to 5.1 & -6.0 to 24.0 \\
\hline \multicolumn{4}{|c|}{ Total } & 54 & 46 & $94 \mathrm{deg}^{2}$ & & & & & \\
\hline L4h & $13: 35$ & $-09: 00$ & 0.89 & 20 & 16 & $7 \times 2 ; 1 \times 1$ & 2004 Apr 26 & G.MP9401 & 24.06 & 0.8 to 6.0 & 2.0 to 42.0 \\
\hline $\mathrm{L} 4 \mathrm{j}$ & $15: 12$ & $-16: 51$ & 0.89 & 10 & 10 & $8 \times 2$ & 2004 Apr 25 & G.MP9401 & 24.00 & 0.8 to 5.6 & -3.6 to 36.4 \\
\hline L4k & $15: 12$ & $-18: 47$ & 0.90 & 19 & 16 & $8 \times 2$ & 2004 May 24 & G.MP9401 & 24.35 & 0.8 to 5.7 & -1.0 to 35.0 \\
\hline $\mathrm{L} 4 \mathrm{~m}$ & 19:14 & $-22: 47$ & 0.89 & 4 & 4 & $12 \times 1$ & 2004 Jun 25 & G.MP9401 & 23.76 & 0.8 to 5.6 & -25.0 to 15.0 \\
\hline $\mathrm{L} 4 \mathrm{n}$ & $19: 23$ & $-21: 33$ & 0.90 & 4 & 4 & $14 \times 1$ & 2004 Jul 22 & G.MP9401 & 23.74 & 0.8 to 6.0 & -27.7 to 12.3 \\
\hline L4o & $19: 15$ & $-23: 46$ & 0.90 & 2 & 1 & $13 \times 1$ & 2004 Jul 24 & G.MP9401 & 23.53 & 0.8 to 6.0 & -24.7 to 11.3 \\
\hline L4p & $20: 53$ & $-18: 27$ & 0.85 & 9 & 9 & $8 \times 2$ & 2004 Aug 15 & G.MP9401 & 24.00 & 1.0 to 5.7 & -30.0 to 0.0 \\
\hline $\mathrm{L} 4 \mathrm{q}$ & $21: 26$ & $-16: 05$ & 0.85 & 14 & 10 & $8 \times 2$ & 2004 Aug 19 & G.MP9401 & 24.21 & 1.2 to 6.1 & -35.5 to -0.5 \\
\hline $\mathrm{L} 4 \mathrm{v}$ & $02: 35$ & $15: 10$ & 0.78 & 18 & 14 & $2 \times 2 ; 1 \times 1 ; 5 \times 2$ & 2004 Nov 9 & G.MP9401 & 24.40 & 0.8 to 6.3 & -34.0 to -2.0 \\
\hline \multicolumn{4}{|c|}{ Total } & 100 & 84 & $133 \mathrm{deg}^{2}$ & & & & & \\
\hline L5c & 09:11 & $17: 13$ & 0.84 & 21 & 19 & $7 \times 2 ; 1 \times 1$ & 2005 Feb 10 & G.MP9401 & 24.30 & 0.8 to 6.4 & -1.0 to 31.0 \\
\hline L5i & $16: 18$ & $-22: 18$ & 0.90 & 7 & 7 & $8 \times 2$ & 2005 May 12 & G.MP9401 & 23.84 & 0.4 to 7.3 & -9.4 to 32.2 \\
\hline $\mathrm{L} 5 \mathrm{j}$ & $16: 09$ & $-19: 59$ & 0.89 & 3 & 3 & $8 \times 2$ & 2005 Jun 10 & G.MP9401 & 23.49 & 0.4 to 7.0 & -9.9 to 33.9 \\
\hline L5r & $22: 36$ & $03: 55$ & 0.90 & 1 & 1 & $3 \times 2 ; 1 \times 1 ; 2 \times 2$ & 2005 Sep 3 & G.MP9401 & 23.89 & 0.7 to 7.5 & -42.1 to -1.9 \\
\hline L5s & $22: 28$ & $14: 35$ & 0.90 & 1 & 1 & $3 \times 2 ; 1 \times 1 ; 2 \times 2$ & 2005 Sep 3 & G.MP9401 & 24.00 & 0.7 to 7.5 & -41.8 to -2.0 \\
\hline $\mathrm{L} 7 \mathrm{a}$ & $08: 43$ & $18: 30$ & 0.89 & 9 & 8 & patchy & 2007 Jan 19 & G.MP9401 & 23.98 & 0.8 to 7.7 & -4.1 to 34.9 \\
\hline \multicolumn{4}{|c|}{ Total } & 42 & 39 & $94 \mathrm{deg}^{2}$ & & & & & \\
\hline \multicolumn{4}{|c|}{ Grand Total } & 196 & 169 & $321 \mathrm{deg}^{2}$ & & & & & \\
\hline Pre & $22: 00$ & $-13: 00$ & 0.90 & 13 & 10 & $3.5 \times 2$ & 2002 Aug 5 & $\mathrm{R}$ & $24.85^{\mathrm{g}}$ & 0.8 to 8.0 & -35.0 to -5.0 \\
\hline
\end{tabular}

Notes.

${ }^{a}$ R.A./decl. is the approximate center of the field.

${ }^{b}$ Fill factor is the fraction of the rectangle covered by the mosaic and useful for TNO searching.

c The number of objects in Columns 5 and 6 correspond to those detected and tracked in the characterized sample, as defined in Section 3.

$\mathrm{d}$ The limiting magnitude of the survey, $g_{A B}$, is in the SDSS photometric system and corresponding to a $40 \%$ efficiency of detection.

e Detection limits give the limits on the sky motion in rate (" $/ \mathrm{hr}$ ) and direction (" 0 deg" is due west and positive to the north).

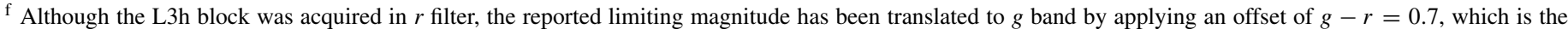
average $g-r$ color of our full sample (see Table 7).

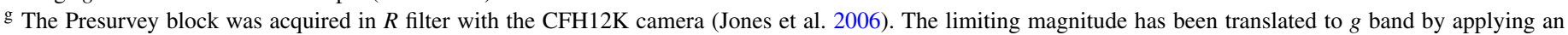
offset of $g-R=0.8$.

an initial data release of the complete observing record for the L3 objects (objects discovered in 2003; before all the refinement observations for all objects were complete). The L3 release was reported to the Minor Planet Center (MPC; Gladman et al. 2006; Kavelaars et al. 2006a, 2006b) and additional follow-up that has occurred since the 2006 release has also been reported to the MPC. The final release of the complete observing record for all remaining CFEPS objects is available from the MPC (Kavelaars et al. 2011). Detailed astrometric and photometric data for the CFEPS objects can be found on the CFEPS specific databases. ${ }^{12}$ The correspondence between CFEPS internal designations and MPC designations can be determined using Tables 3 and 4 or from electronic tables on the cfeps.net site. All characterized and tracked objects are prefixed by $L$ and are used with the survey simulator for our modeling below. The tracking observations provide sufficient information to allow reliable orbits to be determined such that unambiguous dynamical classification can be achieved in nearly all cases. Ephemeris errors are smaller than a few tens of arcseconds over the next five years. Our standard was to pursue tracking observations until the semimajor axis uncertainty was $<0.1 \%$; in Tables 3 and 4 , orbital elements are shown to the precision with which they are known, with typical

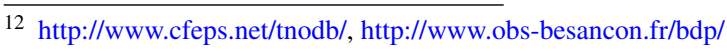

fractional accuracies on the order of $10^{-4}$ or better. In the cases of resonant objects even this precision may not be enough to determine the amplitude of the resonant argument.

Of the 196 TNOs in our CFEPS characterized sample 169 have been tracked through three oppositions or more (i.e., not lost) and their orbits are now known to a precision of $\Delta a / a<$ $0.1 \%$ and can be reliably classified into orbital sub-populations (see below). The very high fraction of our characterized sample for which classification is possible (86\%) is by far the largest "tracking fraction" among large-scale TNO surveys to date and is due to the strong emphasis on follow-up observations in our observing strategy, made possible thanks to the time allocation committees of the many observatories listed in Table 2.

The initial tracking of TNOs discovered by CFEPS is through blind return to the discovery fields to ensure that there is no orbital bias in the tracked fraction. We do find, however, that the tracked fraction is a function of the magnitude of the TNO and have characterized this bias. For the full CFEPS fields we find the same magnitude dependence as for the L3 fields for objects brighter than the limit of the characterized sample, which we model as

$$
f_{t, L 7}(g)= \begin{cases}1.0 & (g \leqslant 22.8) \\ 1.0-0.25(g-22.8) & (g>22.8),\end{cases}
$$


Table 2

Follow-up/Tracking Observations

\begin{tabular}{|c|c|c|}
\hline UT Date & Telescope & No. Obs. \\
\hline 2002 Aug 5 & $\mathrm{CFHT}+12 \mathrm{k}$ & 6 \\
\hline 2002 Sep 3 & NOT $2.56 \mathrm{~m}$ & 6 \\
\hline 2002 Sep 2 & Calar-Alto $2.2 \mathrm{~m}$ & 9 \\
\hline 2002 Sep 30 & CFHT $3.5 \mathrm{~m}$ & 6 \\
\hline 2002 Nov 28 & CFHT $3.5 \mathrm{~m}$ & 10 \\
\hline 2003 Jul 26 & $\mathrm{ESO} 2.2 \mathrm{~m}$ & 6 \\
\hline 2004 Feb 19 & WIYN $3.5 \mathrm{~m}$ & 4 \\
\hline 2004 Apr 15 & Hale $5 \mathrm{~m}$ & 73 \\
\hline 2004 May 24 & Mayall $3.8 \mathrm{~m}$ & 6 \\
\hline 2004 Aug 12 & CFHT $3.5 \mathrm{~m}$ & 15 \\
\hline 2004 Sep 6 & KPNO 2 m & 15 \\
\hline 2004 Sep 11 & Mayall $3.8 \mathrm{~m}$ & 25 \\
\hline 2004 Sep 16 & Hale $5 \mathrm{~m}$ & 20 \\
\hline 2004 Sep 21 & CFHT $3.5 \mathrm{~m}$ & 4 \\
\hline $2005 \mathrm{Jul} 8$ & Gemini-North $8 \mathrm{~m}$ & 45 \\
\hline $2005 \mathrm{Jul} 9$ & Hale $5 \mathrm{~m}$ & 47 \\
\hline 2005 Jul 11 & ESO $2.2 \mathrm{~m}$ & 25 \\
\hline 2005 Aug 1 & VLT UT-1 & 53 \\
\hline 2005 Sep 24 & WIYN $3.5 \mathrm{~m}$ & 9 \\
\hline 2005 Oct 3 & Hale $5 \mathrm{~m}$ & 72 \\
\hline 2005 Nov 4 & Mayall $3.8 \mathrm{~m}$ & 31 \\
\hline 2005 Dec 4 & MDM $2.4 \mathrm{~m}$ & 10 \\
\hline 2006 Jan 28 & Hale $5 \mathrm{~m}$ & 50 \\
\hline 2006 May 1 & CFHT $3.5 \mathrm{~m}$ & 23 \\
\hline 2006 May 2 & WIYN 3.5 m & 32 \\
\hline 2006 May 26 & CFHT $3.5 \mathrm{~m}$ & 20 \\
\hline 2006 Jun 25 & Mayall $3.8 \mathrm{~m}$ & 2 \\
\hline 2006 Jul 3 & CFHT 3.5 m & 18 \\
\hline 2006 Jul 26 & Hale $5 \mathrm{~m}$ & 15 \\
\hline 2006 Sep 18 & CFHT $3.5 \mathrm{~m}$ & 7 \\
\hline 2006 Sep 26 & MMT $6.5 \mathrm{~m}$ & 11 \\
\hline 2006 Oct 22 & Hale $5 \mathrm{~m}$ & 29 \\
\hline 2006 Oct 21 & WHT 4 m & 17 \\
\hline 2006 Nov 23 & WIYN $3.5 \mathrm{~m}$ & 41 \\
\hline 2007 Feb 14 & $2.1 \mathrm{~m}$ reflector & 3 \\
\hline 2007 Feb 21 & Hale $5 \mathrm{~m}$ & 22 \\
\hline 2007 May 15 & Hale $5 \mathrm{~m}$ & 45 \\
\hline 2007 May 15 & KPNO 2 m & 23 \\
\hline 2007 Jun 2 & MMT $6.5 \mathrm{~m}$ & 3 \\
\hline 2007 Sep 11 & WIYN $3.5 \mathrm{~m}$ & 32 \\
\hline 2007 Sep 16 & Hale $5 \mathrm{~m}$ & 27 \\
\hline 2007 Nov 8 & WIYN $3.5 \mathrm{~m}$ & 30 \\
\hline 2008 May 3 & WIYN 3.5 m & 52 \\
\hline 2008 Jun 7 & CTIO 4 m & 28 \\
\hline 2008 Oct 23 & WIYN $3.5 \mathrm{~m}$ & 3 \\
\hline 2008 Dec 6 & Hale $5 \mathrm{~m}$ & 9 \\
\hline 2009 Jan 26 & CFHT $3.5 \mathrm{~m}$ & 19 \\
\hline 2009 Apr 17 & MMT $6.5 \mathrm{~m}$ & 3 \\
\hline 2009 Apr 23 & Subaru $8 \mathrm{~m}$ & 1 \\
\hline 2009 Jun 20 & WIYN $3.5 \mathrm{~m}$ & 22 \\
\hline
\end{tabular}

Notes. UT Date is the start of the observing run; No. Obs. is the number of astrometric measures reported from the observing run. Only observations not part of the Very Wide component of CFHT-LS are reported here. Runs with low numbers of astrometric measures were either wiped out by poor weather or not meant for CFEPS objects follow-up originally.

where $f_{t, L 7}$ is the tracked fraction. The tracked fraction remains well above $50 \%$ down to the characterized limit of the survey blocks. We have also re-examined the magnitude dependence of the tracked fraction of our pre-survey discoveries (Jones et al. 2006) and find

$$
f_{t, L 7}(g)= \begin{cases}1.0 & (g \leqslant 24.1) \\ 1.0-2.5(g-24.1) & (g>24.1)\end{cases}
$$

The pre-survey observations used much longer exposure times than for CFEPS, hence the deeper limiting magnitude reached. We also had a smaller survey area and were able to perform a more thorough follow-up campaign, resulting in a tracking efficiency that essentially was $100 \%$ up to the limiting magnitude of the discoveries. Our pre-survey discovery observations were reported on the Landolt-R system and we have transformed our pre-survey limits to $g$, for use in our survey simulator, using a constant color offset of $(g-R)=0.8$ (Hainaut \& Delsanti 2002).

\subsection{Orbit Classification}

We adopt the convention that, based on orbital elements and dynamical behavior, the Kuiper Belt can be divided into three broad orbital classes. An object is checked against each dynamical class in the order below to decide whether or not it belongs to that class, each object can belong to only one class. A schematic representation of this dynamical classification is shown in Figure 1 of Gladman et al. (2008).

1. Resonant (objects currently in an MMR with Neptune).

2. Scattering (objects which over $10 \mathrm{Myr}$ forward in time integrations experience encounters with Neptune resulting in a variation of semimajor axis of more than $1.5 \mathrm{AU}$ ).

3. Classical or detached belt (everything that remains). One further sub-divides the classical belt into:

(a) inner classical belt (objects with semimajor axis interior to the 3:2 MMR),

(b) main classical belt (objects whose semimajor axis is between the 3:2 and 2:1 MMRs),

(c) outer classical belt (objects with semimajor axis exterior to the 2:1 MMR with $e<0.24$ ),

(d) detached (those objects with semimajor axis beyond the 2:1 MMR that have $e>0.24$ ).

The classical belt is often also divided into high-inclination and low-inclination objects. For the L7 model, we work from the hypothesis described in Brown (2001) that two distinct populations exist, one with a wide inclination distribution (the "hot" population) and the other one with a narrow inclination distribution (the "cold" population), with both populations overlapping with each other in inclination space (thus some "cold" objects may have large inclination and some "hot" objects may have low inclination). In the literature, the separation between hot and cold populations is sometimes presented as a sharp cut in inclination, often around $5^{\circ}$, under the assumption that an object with inclination less (greater) than that threshold has a very high likelihood to be a member of the cold (hot) population. As will be seen in Section 5.1.1, the veracity of this assumption depends on the physical size of the objects being sorted, with larger objects $(H<7)$ having a much higher probability of being from the hot population, regardless of their inclination, while the objects from the cold population dominate at smaller $(H>8)$ sizes. A strict inclination cut does not isolate the two mixed populations.

Following the procedure in Gladman et al. (2008) (similar to Chiang et al. 2003a), we extend the L3 sample classification given in P1 to our full CFEPS sample as of 2009 November (including all refinement observations to that date). Using this classification procedure, 15 of our objects remain insecure (even though these have observational arcs extending across five oppositions); all of these are due to their proximity to a resonance border where the remaining astrometric uncertainty makes it unclear if the object is actually resonant. We list these "insecure" 
Table 3

Characterized Object Classification

\begin{tabular}{|c|c|c|c|c|c|c|c|}
\hline CFEPS & MPC Designations & $\begin{array}{c}a \\
(\mathrm{AU})\end{array}$ & $e$ & $\begin{array}{c}i \\
\left({ }^{\circ}\right)\end{array}$ & $\begin{array}{l}\text { Dist. } \\
\text { (AU) }\end{array}$ & \multicolumn{2}{|c|}{ Comment } \\
\hline \multicolumn{8}{|c|}{ Resonant objects } \\
\hline L3y11 & (131697) 2001 XH255 & 34.925 & 0.0736 & 2.856 & 34.0 & $5: 4$ & $\overline{\mathrm{MPC}_{W}}$ \\
\hline L4h14 & 2004 HM79 & 36.441 & 0.07943 & 1.172 & 38.0 & $4: 3$ & \\
\hline $\mathrm{L} 3 \mathrm{~s} 06$ & (143685) 2003 SS317 & 36.456 & 0.2360 & 5.905 & 28.2 & $4: 3$ & \\
\hline $\mathrm{L} 5 \mathrm{c} 23$ & 2005 CF81 & 36.473 & 0.06353 & 0.405 & 34.4 & $4: 3$ & \\
\hline L7a10 & 2005 GH228 & 36.663 & 0.18814 & 17.151 & 30.6 & $4: 3$ & I \\
\hline L4k11 & 2004 KC19 & 39.258 & 0.23605 & 5.637 & 30.2 & $3: 2$ & \\
\hline L4h15 & 2004 HB79 & 39.260 & 0.22862 & 2.661 & 32.0 & $3: 2$ & \\
\hline $\mathrm{L} 5 \mathrm{c} 11$ & 2005 CD81 & 39.262 & 0.15158 & 21.344 & 45.2 & $3: 2$ & \\
\hline L4h06 & 2004 HY78 & 39.302 & 0.19571 & 12.584 & 31.8 & $3: 2$ & \\
\hline L4v 18 & 2004 VY130 & 39.342 & 0.27616 & 10.203 & 28.5 & $3: 2$ & \\
\hline $\mathrm{L} 4 \mathrm{~m} 02$ & 2004 MS8 & 39.344 & 0.29677 & 12.249 & 27.8 & $3: 2$ & \\
\hline $\mathrm{L} 3 \mathrm{~s} 02$ & 2003 SO317 & 39.346 & 0.2750 & 6.563 & 32.3 & $3: 2$ & \\
\hline L4h09PD & (47932) 2000 GN171 & 39.352 & 0.28120 & 10.815 & 28.5 & $3: 2$ & \\
\hline L3h19 & 2003 HF57 & 39.36 & 0.194 & 1.423 & 32.4 & $3: 2$ & \\
\hline L3w07 & 2003 TH58 & 39.36 & 0.0911 & 27.935 & 35.8 & $3: 2$ & \\
\hline L4h07 & 2004 HA79 & 39.378 & 0.24697 & 22.700 & 38.4 & $3: 2$ & \\
\hline L3h11 & 2003 HA57 & 39.399 & 0.1710 & 27.626 & 32.7 & $3: 2$ & \\
\hline L3w01 & 2005 TV189 & 39.41 & 0.1884 & 34.390 & 32.0 & $3: 2$ & \\
\hline L4j11 & 2004 HX78 & 39.420 & 0.15270 & 16.272 & 33.6 & $3: 2$ & \\
\hline L4v09 & 2004 VX130 & 39.430 & 0.20696 & 5.745 & 34.8 & $3: 2$ & \\
\hline L3h14 & 2003 HD57 & 39.44 & 0.179 & 5.621 & 32.9 & $3: 2$ & \\
\hline $\mathrm{L} 3 \mathrm{~s} 05$ & 2003 SR317 & 39.44 & 0.1667 & 8.348 & 35.5 & $3: 2$ & \\
\hline L4v13 & 2004 VV130 & 39.454 & 0.18827 & 23.924 & 32.8 & $3: 2$ & \\
\hline L4k01 & 2004 KB19 & 39.484 & 0.21859 & 17.156 & 39.5 & $3: 2$ & \\
\hline L3h01 & 2004 FW164 & 39.492 & 0.1575 & 9.114 & 33.3 & $3: 2$ & \\
\hline L5i06PD & $2001 \mathrm{KQ77}$ & 39.505 & 0.15619 & 15.617 & 36.2 & $3: 2$ & \\
\hline L4h10PD & 1995 HM5 & 39.521 & 0.25197 & 4.814 & 31.1 & $3: 2$ & \\
\hline $\mathrm{L} 4 \mathrm{v} 12$ & 2004 VZ130 & 39.551 & 0.28159 & 11.581 & 29.2 & $3: 2$ & \\
\hline L4h08 & 2004 HZ78 & 39.580 & 0.15095 & 13.310 & 34.8 & $3: 2$ & \\
\hline $\mathrm{L} 5 \mathrm{c} 08$ & 2006 CJ69 & 42.183 & 0.22866 & 17.916 & 35.5 & $5: 3$ & \\
\hline L3y06 & 2003 YW179 & 42.193 & 0.1537 & 2.384 & 35.7 & $5: 3$ & \\
\hline L5c13PD & 1999 CX131 & 42.240 & 0.23387 & 9.757 & 41.8 & $5: 3$ & \\
\hline L4v05 & 2004 VE131 & 42.297 & 0.25889 & 5.198 & 39.6 & $5: 3$ & \\
\hline L3y $12 \mathrm{PD}$ & (126154) 2001 YH140 & 42.332 & 0.14043 & 11.078 & 36.4 & $5: 3$ & \\
\hline L4k10 & 2004 KK19 & 42.410 & 0.14391 & 4.485 & 46.0 & $5: 3$ & I \\
\hline L3q08PD & (135742) 2002 PB171 & 43.63 & 0.125 & 5.450 & 40.7 & $7: 4$ & \\
\hline $\mathrm{L} 4 \mathrm{n} 03$ & 2004 OQ15 & 43.646 & 0.12472 & 9.727 & 40.5 & $7: 4$ & \\
\hline $\mathrm{L} 3 \mathrm{w} 03$ & 2003 YJ179 & 43.66 & 0.0794 & 1.446 & 40.3 & $7: 4$ & \\
\hline L4v10 & 2004 VF131 & 43.672 & 0.21492 & 0.816 & 42.0 & $7: 4$ & \\
\hline K02O03 & 2000 OP67 & 43.72 & 0.191 & 0.751 & 39.3 & $7: 4$ & \\
\hline L4h11 & 2004 HN79 & 45.736 & 0.22936 & 11.669 & 37.4 & $15: 8$ & I \\
\hline L4h18 & 2004 HP79 & 47.567 & 0.18250 & 2.253 & 39.5 & $2: 1$ & \\
\hline L4k16 & 2004 KL19 & 47.660 & 0.32262 & 5.732 & 32.3 & $2: 1$ & \\
\hline $\mathrm{L} 4 \mathrm{k} 20$ & 2004 KM19 & 47.720 & 0.29180 & 1.686 & 33.8 & $2: 1$ & \\
\hline $\mathrm{K} 02 \mathrm{O} 12$ & 2002 PU170 & 47.75 & 0.2213 & 1.918 & 47.2 & $2: 1$ & \\
\hline L4v06 & 2004 VK78 & 47.764 & 0.33029 & 1.467 & 32.5 & $2: 1$ & \\
\hline L3y07 & (131696) 2001 XT254 & 52.92 & 0.3221 & 0.518 & 36.6 & $7: 3$ & $\mathrm{MPC}_{W}$ \\
\hline L5c19PD & 2002 CZ248 & 53.039 & 0.38913 & 5.466 & 36.2 & $7: 3$ & \\
\hline $\mathrm{L} 5 \mathrm{c} 12$ & 2002 CY224 & 53.892 & 0.34651 & 15.733 & 36.3 & $12: 5$ & \\
\hline L4j08 & 2004 HO79 & 55.206 & 0.41166 & 5.624 & 37.3 & $5: 2$ & \\
\hline L3f04PD & (60621) 2000 FE8 & 55.29 & 0.4020 & 5.869 & 36.0 & $5: 2$ & \\
\hline L4j06PD & 2002 GP32 & 55.387 & 0.42195 & 1.559 & 32.1 & $5: 2$ & \\
\hline L4k14 & 2004 KZ18 & 55.419 & 0.38191 & 22.645 & 34.4 & $5: 2$ & \\
\hline L4h02PD & 2004 EG96 & 55.550 & 0.42291 & 16.213 & 32.2 & $5: 2$ & \\
\hline L4v08 & 2004 VU130 & 62.194 & 0.42806 & 8.024 & 49.7 & $3: 1$ & \\
\hline L3y02 & 2003 YQ179 & 88.38 & 0.5785 & 20.873 & 39.3 & $5: 1$ & I \\
\hline \multicolumn{8}{|c|}{ Inner classical belt } \\
\hline L3y14PD & (131695) 2001 XS254 & 37.220 & 0.05211 & 4.262 & 35.3 & \multirow{6}{*}{\multicolumn{2}{|c|}{$\mathrm{I}(11: 8)$}} \\
\hline L4q12PD & 2000 OB51 & 37.820 & 0.03501 & 4.458 & 36.6 & & \\
\hline L4q10 & 1999 OJ4 & 38.017 & 0.02539 & 4.000 & 38.1 & & \\
\hline L4k18 & 2004 KD19 & 38.257 & 0.01707 & 2.126 & 38.9 & & \\
\hline $\mathrm{L} 4 \mathrm{o} 01$ & 2004 OP15 & 38.584 & 0.05532 & 22.946 & 38.7 & & \\
\hline L3w06 & 2003 YL179 & 38.82 & 0.002 & 2.525 & 38.7 & & \\
\hline
\end{tabular}


Table 3

(Continued)

\begin{tabular}{|c|c|c|c|c|c|c|}
\hline CFEPS & MPC Designations & $\begin{array}{c}a \\
(\mathrm{AU})\end{array}$ & $e$ & $\begin{array}{c}i \\
\left(^{\circ}\right)\end{array}$ & $\begin{array}{l}\text { Dist. } \\
\text { (AU) }\end{array}$ & Comment \\
\hline \multicolumn{7}{|c|}{ Main classical belt } \\
\hline L4k12 & 2004 KH19 & 40.772 & 0.11721 & 35.230 & 43.6 & \\
\hline L4q05 & 2004 QE29 & 40.878 & 0.08372 & 24.125 & 37.5 & \\
\hline L4k19 & 2005 JB186 & 41.471 & 0.10588 & 20.220 & 38.0 & \\
\hline L3w05 & 2003 YK179 & 41.67 & 0.146 & 19.605 & 42.7 & \\
\hline L4h16 & 2004 HL79 & 42.126 & 0.07520 & 16.759 & 40.0 & \\
\hline L5s01PD & (120347) 2004 SB60 & 42.028 & 0.10667 & 23.931 & 43.7 & \\
\hline $\mathrm{L} 3 \mathrm{~s} 01$ & 2003 SN317 & 42.50 & 0.0421 & 1.497 & 41.5 & \\
\hline L4q15 & 1999 ON4 & 42.571 & 0.03995 & 3.187 & 40.9 & \\
\hline L3h05 & 2003 HY56 & 42.604 & 0.037 & 2.578 & 42.5 & \\
\hline L3q02PD & 2001 QB298 & 42.618 & 0.0962 & 1.800 & 39.1 & \\
\hline $\mathrm{L} 3 \mathrm{~s} 03$ & 2003 SQ317 & 42.63 & 0.0795 & 28.568 & 39.3 & \\
\hline K02O20 & 2002 PV170 & 42.643 & 0.016 & 1.271 & 42.2 & \\
\hline K02P32 & 2002 PX170 & 42.65 & 0.041 & 1.570 & 42.8 & \\
\hline $\mathrm{L} 5 \mathrm{c} 03$ & 2005 CE81 & 42.715 & 0.04666 & 3.084 & 40.8 & \\
\hline L5i01 & 2006 HA123 & 42.778 & 0.04615 & 3.303 & 41.0 & \\
\hline L4p02 & 2004 PU117 & 42.817 & 0.01461 & 1.874 & 42.4 & \\
\hline L4p01 & 2004 PT117 & 42.983 & 0.04115 & 1.238 & 43.6 & \\
\hline K02O40 & 2002 PY170 & 43.015 & 0.030 & 3.016 & 43.0 & \\
\hline $\mathrm{L} 4 \mathrm{q} 03$ & 2004 QD29 & 43.020 & 0.11388 & 23.862 & 40.6 & $\mathrm{I}(12: 7)$ \\
\hline L3w11 & 2003 TK58 & 43.078 & 0.0647 & 3.355 & 45.6 & \\
\hline $\mathrm{L} 4 \mathrm{~m} 03$ & 2004 MT8 & 43.120 & 0.04195 & 2.239 & 44.9 & \\
\hline L4h05PD & 2001 FK185 & 43.255 & 0.03994 & 1.171 & 41.7 & \\
\hline $\mathrm{L} 4 \mathrm{j} 10$ & 2004 HH79 & 43.259 & 0.06010 & 8.610 & 43.2 & \\
\hline $\mathrm{L} 4 \mathrm{j} 02$ & 2004 HF79 & 43.269 & 0.02547 & 1.484 & 42.4 & \\
\hline L4k04 & 2004 KG19 & 43.272 & 0.02164 & 0.963 & 42.4 & \\
\hline L5c07PD & 2005 XU100 & 43.398 & 0.10283 & 7.869 & 41.7 & \\
\hline L7a06 & 2006 WF206 & 43.500 & 0.04246 & 2.056 & 44.4 & \\
\hline L3w10 & 2003 TL58 & 43.542 & 0.0456 & 7.738 & 42.2 & \\
\hline L3y01 & 2003 YX179 & 43.582 & 0.044 & 4.850 & 42.5 & \\
\hline L3y05 & 2003 YS179 & 43.585 & 0.022 & 3.727 & 43.8 & \\
\hline L3h18 & 2003 HG57 & 43.612 & 0.0323 & 2.098 & 43.0 & \\
\hline L4p05 & 2004 PW117 & 43.620 & 0.06023 & 1.862 & 46.0 & \\
\hline $\mathrm{L} 7 \mathrm{a} 05$ & 2005 BV49 & 43.684 & 0.04575 & 7.981 & 41.8 & \\
\hline L5j04 & 2005 LB54 & 43.690 & 0.04752 & 3.006 & 41.8 & \\
\hline L4h01PD & (181708) 1993 FW & 43.717 & 0.04807 & 7.750 & 41.9 & \\
\hline L4p06PD & 2001 QY297 & 43.835 & 0.08332 & 1.547 & 42.8 & \\
\hline L4h12 & 2004 HK79 & 43.888 & 0.07800 & 1.946 & 41.3 & \\
\hline L5i03PD & $2001 \mathrm{KO} 77$ & 43.898 & 0.14569 & 20.726 & 37.7 & \\
\hline L4h13 & 2004 HJ79 & 43.947 & 0.04419 & 3.317 & 45.0 & \\
\hline $\mathrm{L} 4 \mathrm{v} 03$ & $2004 \mathrm{VC} 131$ & 43.951 & 0.07395 & 0.490 & 40.7 & \\
\hline $\mathrm{L} 5 \mathrm{c} 22$ & 2007 DS101 & 43.991 & 0.08474 & 1.389 & 44.6 & \\
\hline L3h13 & 2003 HH57 & 44.04 & 0.088 & 1.436 & 40.2 & \\
\hline L3h09 & 2003 HC57 & 44.05 & 0.072 & 1.038 & 43.4 & \\
\hline L5i05 & 2005 JY185 & 44.077 & 0.06848 & 2.139 & 44.6 & \\
\hline $\mathrm{L} 7 \mathrm{a} 07$ & 2005 BW49 & 44.097 & 0.07959 & 2.102 & 41.9 & \\
\hline L3q06PD & 2001 QJ298 & 44.10 & 0.0388 & 2.151 & 45.2 & \\
\hline $\mathrm{L} 4 \mathrm{k} 03$ & $2004 \mathrm{KF} 19$ & 44.123 & 0.06348 & 0.108 & 41.4 & \\
\hline L5c21PD & 2005 EE296 & 44.126 & 0.06804 & 3.296 & 46.2 & \\
\hline L3q09PD & 2001 QX297 & 44.15 & 0.0275 & 0.911 & 43.5 & \\
\hline L5c18 & 2007 CS79 & 44.159 & 0.03582 & 1.540 & 42.8 & \\
\hline L3h20 & 2003 HE57 & 44.17 & 0.100 & 8.863 & 40.0 & \\
\hline L5c24PD & 1999 CU153 & 44.172 & 0.06520 & 2.698 & 42.7 & \\
\hline $\mathrm{L} 4 \mathrm{v} 02$ & 2004 VB131 & 44.189 & 0.07267 & 1.747 & 46.5 & \\
\hline $\mathrm{L} 4 \mathrm{j} 03$ & 2004 HG79 & 44.200 & 0.02298 & 3.595 & 43.2 & \\
\hline L4p09 & 2004 PX117 & 44.261 & 0.09965 & 3.747 & 46.1 & \\
\hline L4p08PD & 2001 QZ297 & 44.283 & 0.06442 & 1.856 & 42.0 & \\
\hline L5j03 & 2005 LA54 & 44.314 & 0.06719 & 7.919 & 41.6 & \\
\hline $\mathrm{L} 4 \mathrm{j} 01$ & 2004 HE79 & 44.316 & 0.09805 & 3.089 & 40.0 & \\
\hline $\mathrm{K} 02 \mathrm{P} 41$ & 2002 PA171 & 44.34 & 0.076 & 2.511 & 47.7 & \\
\hline $\mathrm{L} 4 \mathrm{k} 02$ & 2004 KE19 & 44.360 & 0.04981 & 1.178 & 42.6 & \\
\hline L3w08 & 2003 TJ58 & 44.40 & 0.0864 & 0.954 & 40.8 & \\
\hline L3w02 & 2003 TG58 & 44.54 & 0.103 & 1.660 & 43.7 & $\mathrm{I}(9: 5)$ \\
\hline L3w04 & (143991) 2003 YO179 & 44.602 & 0.1370 & 19.393 & 41.3 & \\
\hline L5i08 & $2005 \mathrm{JJ} 186$ & 44.636 & 0.09431 & 4.141 & 41.8 & \\
\hline
\end{tabular}


Table 3

(Continued)

\begin{tabular}{|c|c|c|c|c|c|c|}
\hline CFEPS & MPC Designations & $\begin{array}{c}a \\
(\mathrm{AU})\end{array}$ & $e$ & $\begin{array}{c}i \\
\left({ }^{\circ}\right)\end{array}$ & $\begin{array}{l}\text { Dist. } \\
(\mathrm{AU})\end{array}$ & Comment \\
\hline $\mathrm{K} 02 \mathrm{O} 43$ & 2002 PC171 & 44.706 & 0.059 & 3.574 & 42.7 & \\
\hline L4n04 & 2004 MU8 & 44.856 & 0.08180 & 3.580 & 48.2 & \\
\hline K02O32 & 2002 PW170 & 44.88 & 0.074 & 3.933 & 47.4 & \\
\hline L4q16 & (66452) 1999 OF4 & 44.933 & 0.06380 & 2.660 & 45.2 & \\
\hline $\mathrm{L} 4 \mathrm{j} 12$ & 2006 JV58 & 44.961 & 0.06094 & 0.317 & 42.2 & \\
\hline L5c20PD & 2002 CZ224 & 44.980 & 0.06304 & 1.687 & 47.7 & I $(11: 6)$ \\
\hline L3w09 & 2004 XX190 & 45.171 & 0.1042 & 1.577 & 40.9 & \\
\hline L5c10PD & 1999 CJ119 & 45.325 & 0.06651 & 3.205 & 42.3 & \\
\hline L5c06 & 2007 CQ79 & 45.441 & 0.07721 & 1.185 & 45.8 & \\
\hline L4v01 & 2004 VA131 & 45.538 & 0.09613 & 0.767 & 41.2 & \\
\hline L4k15PD & 2003 LB7 & 45.580 & 0.13130 & 2.294 & 40.1 & \\
\hline $\mathrm{L} 5 \mathrm{c} 02$ & 2006 CH69 & 45.735 & 0.03535 & 1.791 & 44.2 & \\
\hline L4q11 & 1999 OM4 & 45.924 & 0.11643 & 2.088 & 44.0 & \\
\hline L4j07 & 2004 HD79 & 45.941 & 0.03205 & 1.305 & 47.3 & \\
\hline L5i02PD & $2001 \mathrm{KW} 76$ & 46.013 & 0.21613 & 10.460 & 39.6 & \\
\hline L4p03 & 2004 PV117 & 46.069 & 0.15343 & 4.324 & 39.5 & \\
\hline L7a04PD & 2002 CY248 & 46.191 & 0.14635 & 7.038 & 51.8 & \\
\hline $\mathrm{L} 4 \mathrm{k} 13$ & 2006 JU58 & 46.239 & 0.12464 & 7.035 & 46.5 & \\
\hline L3q04PD & 2002 PT170 & 46.24 & 0.143 & 3.703 & 50.5 & \\
\hline L4v14 & 2004 VD131 & 46.324 & 0.12253 & 3.646 & 41.5 & \\
\hline L4j05 & 2004 HC79 & 46.399 & 0.16064 & 1.446 & 39.0 & \\
\hline L4q09 & 2000 PD30 & 46.519 & 0.02232 & 4.594 & 45.7 & \\
\hline L3y03 & 2003 YU179 & 46.75 & 0.1597 & 4.855 & 39.6 & \\
\hline L4k17 & 2004 KJ19 & 46.967 & 0.23543 & 24.421 & 38.5 & \\
\hline L7a11PD & $2000 \mathrm{CO} 105$ & 47.046 & 0.14750 & 19.270 & 49.3 & \\
\hline L3y09 & 2003 YV179 & 47.10 & 0.222 & 15.569 & 41.1 & \\
\hline $\mathrm{L} 5 \mathrm{c} 14$ & 2007 CR79 & 47.149 & 0.21876 & 21.869 & 36.9 & \\
\hline L3h04 & 2003 HX56 & 47.196 & 0.2239 & 29.525 & 45.5 & \\
\hline L4m04 & 2004 MV8 & 47.234 & 0.17503 & 27.205 & 39.1 & \\
\hline \multicolumn{7}{|c|}{ Outer classical belt } \\
\hline L4q06 & 2004 QG29 & 48.480 & 0.23517 & 27.134 & 37.8 & \\
\hline L4q14 & 2004 QH29 & 50.859 & 0.22922 & 12.010 & 39.9 & \\
\hline L5c16 & 2005 CG81 & 53.834 & 0.23684 & 26.154 & 44.6 & \\
\hline \multicolumn{7}{|c|}{ Detached classical belt } \\
\hline L5i04 & 2005 JK186 & 47.264 & 0.24363 & 27.252 & 38.1 & \\
\hline $\mathrm{L} 3 \mathrm{q} 03$ & 2003 QX113 & 49.55 & 0.252 & 6.753 & 58.3 & \\
\hline $\mathrm{L} 7 \mathrm{a} 02$ & 2006 WG206 & 50.416 & 0.29111 & 14.297 & 38.9 & \\
\hline L4p04PD & 2000 PE30 & 54.318 & 0.34216 & 18.416 & 37.6 & \\
\hline L5c15 & 2005 CH81 & 55.156 & 0.31812 & 5.136 & 37.6 & \\
\hline L4n06 & 2004 OS15 & 55.760 & 0.31667 & 4.248 & 39.5 & \\
\hline $\mathrm{L} 4 \mathrm{n} 05$ & 2004 OR15 & 56.248 & 0.33882 & 6.919 & 37.3 & \\
\hline L3f01 & 2003 FZ129 & 61.71 & 0.3840 & 5.793 & 38.0 & \\
\hline $\mathrm{L} 4 \mathrm{~h} 21$ & 2004 HQ79 & 63.299 & 0.42264 & 6.473 & 36.6 & \\
\hline L5j02 & 2005 LC54 & 67.354 & 0.46279 & 22.443 & 43.1 & I (10:3) \\
\hline L5r01 & 2005 RH52 & 153.800 & 0.74644 & 20.447 & 39.0 & I scattering \\
\hline \multicolumn{7}{|c|}{ Scattering disk } \\
\hline L4k09 & 2004 KV18 & 30.192 & 0.18517 & 13.586 & 26.6 & \\
\hline L4m01 & 2004 MW8 & 33.479 & 0.33308 & 8.205 & 31.4 & \\
\hline L4p07 & 2004 PY117 & 39.953 & 0.28088 & 23.545 & 29.6 & \\
\hline $\mathrm{L} 3 \mathrm{q} 01$ & 2003 QW113 & 50.99 & 0.484 & 6.922 & 38.2 & \\
\hline $\mathrm{L} 7 \mathrm{a} 03$ & 2006 BS284 & 59.613 & 0.43949 & 4.575 & 47.0 & \\
\hline L4v11 & 2004 VH131 & 60.036 & 0.62928 & 11.972 & 26.8 & \\
\hline L4v04 & 2004 VG131 & 64.100 & 0.50638 & 13.642 & 31.8 & \\
\hline L3h08 & 2003 HB57 & 159.6 & 0.7613 & 15.499 & 38.4 & \\
\hline
\end{tabular}

Notes. M:N: object in the M:N resonance; I: indicates that the orbit classification is insecure (see Gladman et al. (2008) for an explanation of the exact meaning); (M:N): object may be in the M:N resonance; $\mathrm{MPC}_{W}$ : indicates object was in MPC database but found $+1^{\circ}$ from predicted location. Objects prefixed with $L$ are the characterized, tracked objects discovered during CFEPS; objects prefixed with KO2 were discovered in our pre-survey (Jones et al. 2006); The full orbital elements are available in electronic form from either http://www.cfeps.net/tnodb/ or the MPC. 
Table 4

Non-characterized Object Classification

\begin{tabular}{|c|c|c|c|c|c|c|}
\hline CFEPS & MPC Designations & $\begin{array}{c}a \\
(\mathrm{AU})\end{array}$ & $e$ & $\begin{array}{c}i \\
\left({ }^{\circ}\right)\end{array}$ & $\begin{array}{l}\text { Dist. } \\
\text { (AU) }\end{array}$ & Comment \\
\hline \multicolumn{7}{|c|}{ Resonant Objects } \\
\hline U5j06 & & 39.369 & 0.22055 & 13.525 & 31.2 & $3: 2$ \\
\hline $\mathrm{U} 3 \mathrm{~s} 04$ & 2003 SP317 & 45.961 & 0.1694 & 5.080 & 44.9 & $17: 9 \quad \mathrm{I}$ \\
\hline $\mathrm{U} 7 \mathrm{a} 08$ & & 47.702 & 0.19600 & 7.020 & 38.4 & $2: 1$ \\
\hline U5j01PD & (136120) 2003 LG7 & 62.157 & 0.47825 & 20.104 & 33.1 & $3: 1$ \\
\hline \multicolumn{7}{|c|}{ Main classical belt } \\
\hline U3w13 & 2003 YM179 & 40.960 & 0.056 & 23.414 & 40.2 & \\
\hline U3f02 & 2003 FA130 & 42.602 & 0.031 & 0.288 & 41.3 & \\
\hline U4j09 & & 42.642 & 0.00775 & 3.044 & 42.3 & \\
\hline $\mathrm{U} 7 \mathrm{a} 09$ & & 42.701 & 0.09231 & 2.931 & 44.8 & \\
\hline U3w17 & 2002 WL21 & 43.103 & 0.0415 & 2.552 & 41.6 & \\
\hline U3y 16 & 2003 YR179 & 43.421 & 0.0523 & 9.823 & 41.3 & \\
\hline U3y04 & 2003 YT179 & 43.542 & 0.028 & 1.684 & 44.4 & \\
\hline U3h06 & 2003 HZ56 & 43.63 & 0.010 & 2.550 & 43.5 & \\
\hline U5c17PD & 1999 CN119 & 43.733 & 0.04043 & 0.999 & 44.5 & \\
\hline $\mathrm{U} 4 \mathrm{n} 01$ & & 43.915 & 0.13500 & 0.271 & 43.7 & \\
\hline U3y08 & 2003 YP179 & 44.03 & 0.079 & 0.947 & 41.3 & \\
\hline $\mathrm{U} 4 \mathrm{n} 02$ & & 44.056 & 0.06176 & 2.943 & 46.8 & \\
\hline $\mathrm{U} 3 \mathrm{w} 16$ & 2003 YN179 & 44.272 & 0.006 & 2.768 & 44.4 & \\
\hline U4j04PD & 2000 JF81 & 46.117 & 0.10218 & 1.742 & 44.9 & \\
\hline \multicolumn{7}{|c|}{ Scattering disk } \\
\hline$\overline{\mathrm{U} 7 \mathrm{a} 01}$ & & 42.621 & 0.16444 & 4.742 & 38.9 & I (5:3) \\
\hline
\end{tabular}

Notes. M : N: object in the M:N resonance; I: indicates that the orbit classification is insecure (see Gladman et al. (2008) for an explanation of the exact meaning).

objects in the category shown by two of the three clones. Table 3 gives the classification of all characterized objects used for comparison with the Survey Simulator's artificial detections. Several objects had been independently discovered before we submitted our observations to the MPC and are marked with a PD suffix. Although we do not claim "discoverer credit" for these objects, they have just as much scientficially exploitable value because they were detected during our characterized observations and hence can to be included when running our survey simulator. Table 4 gives the classification of the tracked objects below the $40 \%$ efficiency threshold, hence deemed non-characterized and not used in our Survey Simulator comparisons.

One hundred and twenty one (64\%) of the tracked sample are in the classical belt, split into $6(3 \%)$ inner, $101(54 \%)$ main, $3(2 \%)$ outer, and $11(6 \%)$ detached belt objects. Orbital integration shows that $58(31 \%)$ objects are in an MMR with Neptune, 25 (13\%) of which are plutinos. The remaining sample consists of nine (5\%) objects on scattering orbits.

The apparent motion of TNOs in our opposition discovery fields is approximately $\theta\left({ }^{\prime \prime} / \mathrm{hr}\right) \simeq(147 \mathrm{AU}) / r$, where $r$ is the heliocentric distance in AU. With a typical seeing of $0.7-0.9$ arcsecond and a time base of 70-90 minutes between first and third frames, we were sensitive to objects as distant as $r \simeq 125 \mathrm{AU}$, provided they are large enough to be above our flux limit. The furthest object discovered in CFEPS lies at 58.3 AU from the Sun $\left(\mathrm{L} 3 \mathrm{q} 03=2003 \mathrm{QX}_{113}\right.$, a detached object with $a=49.55 \mathrm{AU})$. The short exposure times used (70-90 s) allowed us to detect objects as close as $15 \mathrm{AU}$ without trailing. We elected to use a rate of motion cut corresponding to objects further than $20 \mathrm{AU}$ from Earth.

In the following sections, we present a parameterization of the intrinsic classical Kuiper Belt and scattering disk population implied by our observations. The differing detectability of these populations, in a flux-limited survey, implies that the intrinsic population ratios will be different from the observed ones. We present the more complex analysis of the resonant populations in a companion paper (Gladman et al. 2011).

\section{THE CLASSICAL BELT's ORBITAL DISTRIBUTION}

This section presents the results of our search for an empirical parameterized orbit distribution for the various components of the so-called classical belt. For each sub-component we start with a simple parameterization of the intrinsic orbit and absolute magnitude distributions. We then use the CFEPS Survey Simulator ${ }^{13}$ to determine which members of the intrinsic population would have been detected by the survey. The orbitalelement distributions of the simulated detections are then compared to our characterized sample. This process is iterated with models of increasing complexity until arriving at a model that provides a statistically acceptable match; no cosmogonic considerations are invoked.

Our model search process provided acceptable parameterizations of the main classical belt, the inner classical belt, and the outer+detached population. Our goal is to discover the main features of the orbital distribution and provide a population estimate for each orbital sub-component. While our success in finding acceptable models is not a proof of model uniqueness, we were surprised, in many cases, by the restricted range of acceptable models.

To evaluate a model's quality, we extend the method defined in P1 to more variables. We compute the Anderson-Darling (AD) (Information Technology Laboratory 2011) statistic for the distributions of the orbital elements $a, e, i$, and $q$, and 13 The survey simulator is available online, with all information needed to use
it, at http://www.cfeps.net as a stand-alone package. 


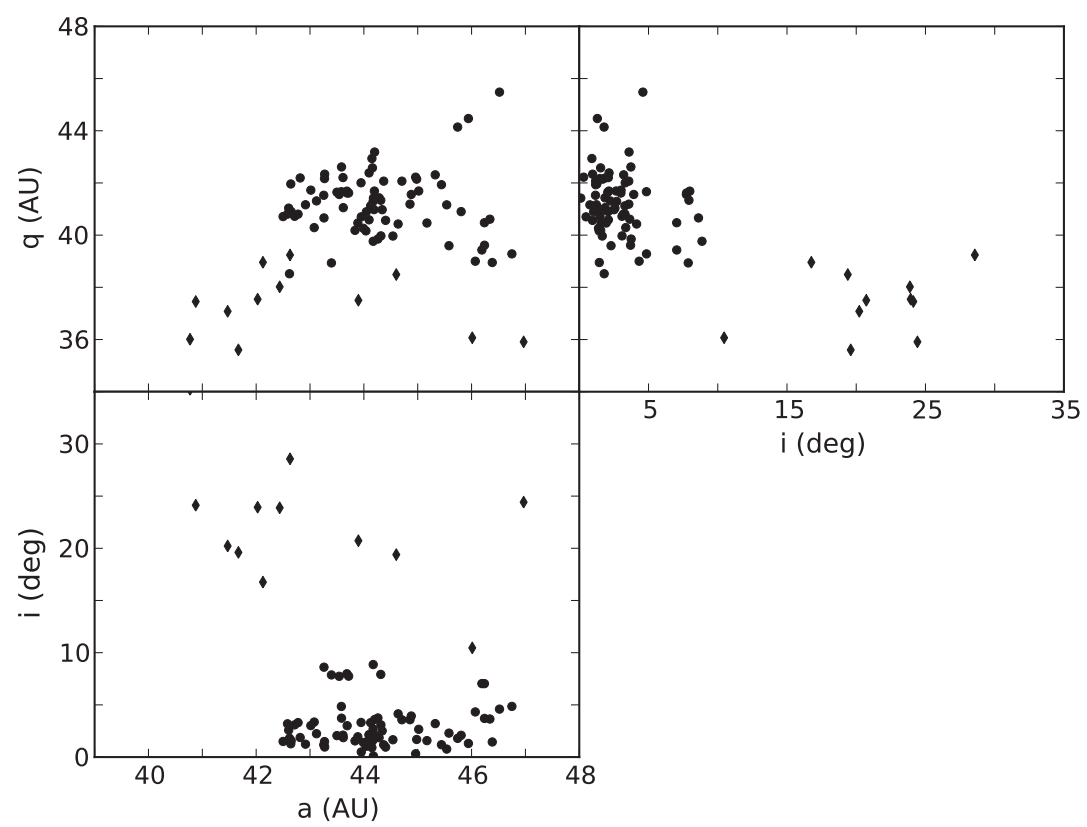

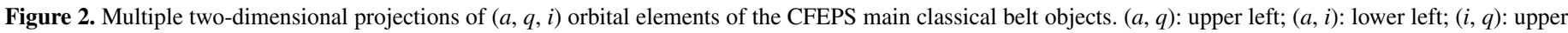

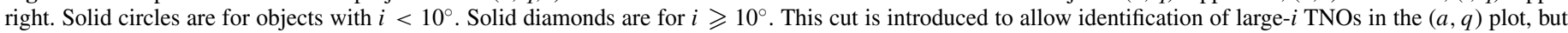
has no relevance to our model.

for $r$ (heliocentric distance at discovery) and $g$ magnitude. We use Kuiper's modified Kolmogorov-Smirnov statistic for the mean anomaly $M$. We follow the same procedure as used in P1 to determine the significance of the computed statistics. For each model parameterization we use the Survey Simulator to draw a large "parent" population from the model. We then draw sub-samples with the same total number as in our L7 characterized sample. Using this simulated "observed" population we compute the various statistics that result from comparing to our large "parent" population. This re-sampling is repeated 5000 times providing a distribution of statistic values for the given parameterization, i.e., "bootstrapping" the statistic. The probability of the statistic measured for the L7 sample is determined by comparing that statistic value to the range of statistic values returned by the bootstrap process. We reject a model if the minimum statistical probability determined in this way is returned by fewer than $5 \%$ of the model bootstraps.

\subsection{The Main Classical Belt}

In P1 we presented a model that matched the orbital distribution of the main classical belt objects detected in the L3 sample; due to the smaller number of objects in the L3 sample, we restricted ourselves to fitting only selected orbital elements and considered a constrained range of the phase-space volume available to main classical belt objects. In addition, P1 did not attempt to determine the absolute magnitude distribution using our detections but instead utilized values available in the literature. Here we restrict our main classical belt model to the $40 \mathrm{AU} \leqslant a \leqslant 47$ AU range, to avoid the complex borders of the 3:2 and 2:1 MMR regions, which includes 88 characterized CFEPS TNOs. This sample size allowed us to remove external constraints on the magnitude distribution and explore a more complete model of the available phase space.

Figures 2 and 3 present $(a, i),(a, q)$, and $(i, q)$ projections of the main-belt TNO orbital elements for characterized CFEPS detections and multi-opposition orbits in the MPC. These figures make it clear that objects with $q<39$ AU are dominantly from the high-inclination population, as was already apparent in the L3 model. The distribution of low- $i$ objects, which span a narrower range of semimajor axis than their high- $i$ cousins, exhibits considerable phase space structure. In an effort to find a parameterization that yielded these interesting sub-structures we investigated a substantial range of empirical representations. We were, however, unable to find a two-component model (like that in P1) that sufficiently reproduced structure observed in the current sample. A more complex representation is required.

After much effort we arrived at our "L7 model" (based on CFEPS discoveries up to mid-2007). The L7 model is composed of three components (Figure 4), the fine details of which are presented in Appendix A. These components are a population with a wide inclination distribution (the hot population) superposed on top of a population with a narrow inclination component with two semimajor axis/eccentricity distributions (the stirred and kernel populations). The hot population is defined as a band in perihelion distance $q$ essentially confined to the range 35-40 AU, with soft exponential decay outside this range. Using a "core" (Elliot et al. 2005) definition based only on inclination does not take into account the transition in the $e / i$ distribution beyond $a \simeq 44.4$ AU clearly visible in both Figures 2 and 3 . With the qualifier that there will be mixing from the low- $i$ tail from the hot component, we thus split the "cold" population of the main classical belt into two subcomponents. The stirred population has orbits drawn from a narrow-inclination distribution with semimajor axes starting at $a=42.5 \mathrm{AU}$ and extending to $a \simeq 47 \mathrm{AU}$, with a range of eccentricities that increases as one goes to larger $a$. The stirred component does not contain the sharp density change at $a \simeq 44.5$ AU. There are more low- $i$ and moderate- $e$ TNOs per unit semimajor axis at $a \sim 44-44.5 \mathrm{AU}$ than at smaller and larger semimajor axes, indicating that a third component is required. To model this component we insert a dense low-inclination concentration, which we call the kernel, near $a=44$ AU to account for this intrinsic population.

The kernel may be the same as the clustering in the $a=$ 42-44 region seen as far back as Jewitt \& Luu (1995) and Jewitt et al. (1996). This also appears to be the same structure 


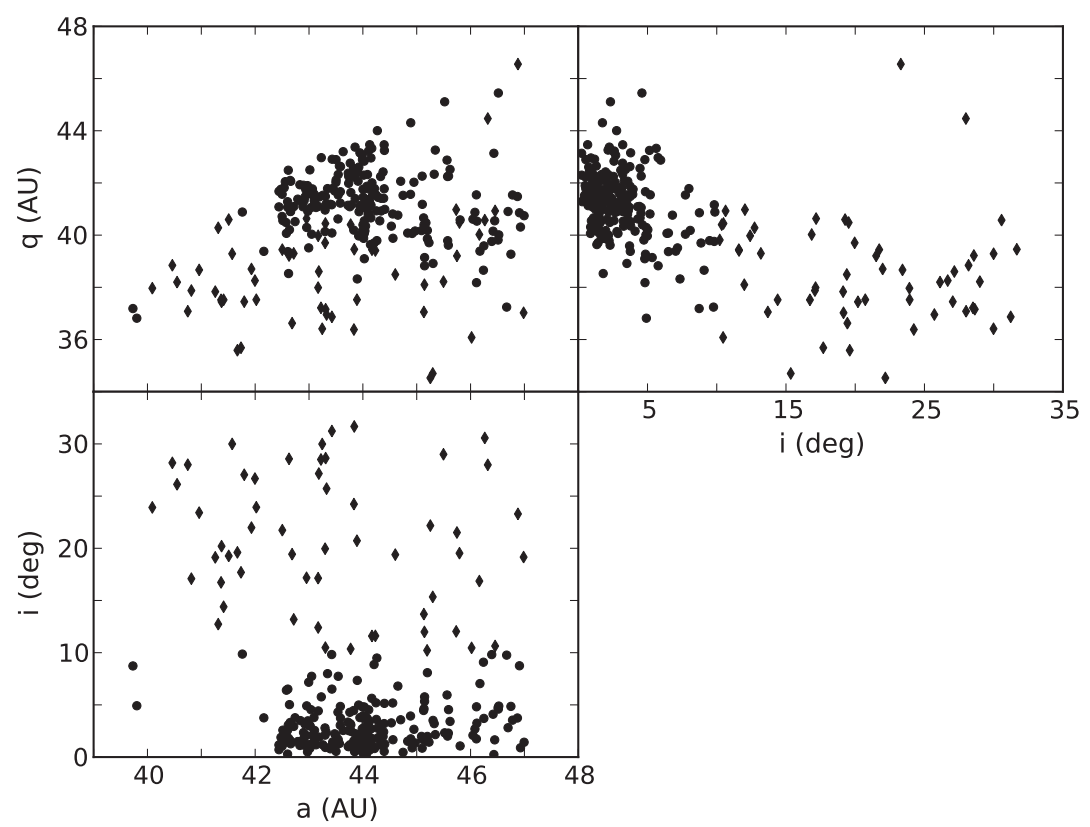

Figure 3. Same as Figure 2 but for the MPC main classical belt objects. L3 and pre-survey objects are present on both plots, as well as any "PD" object.

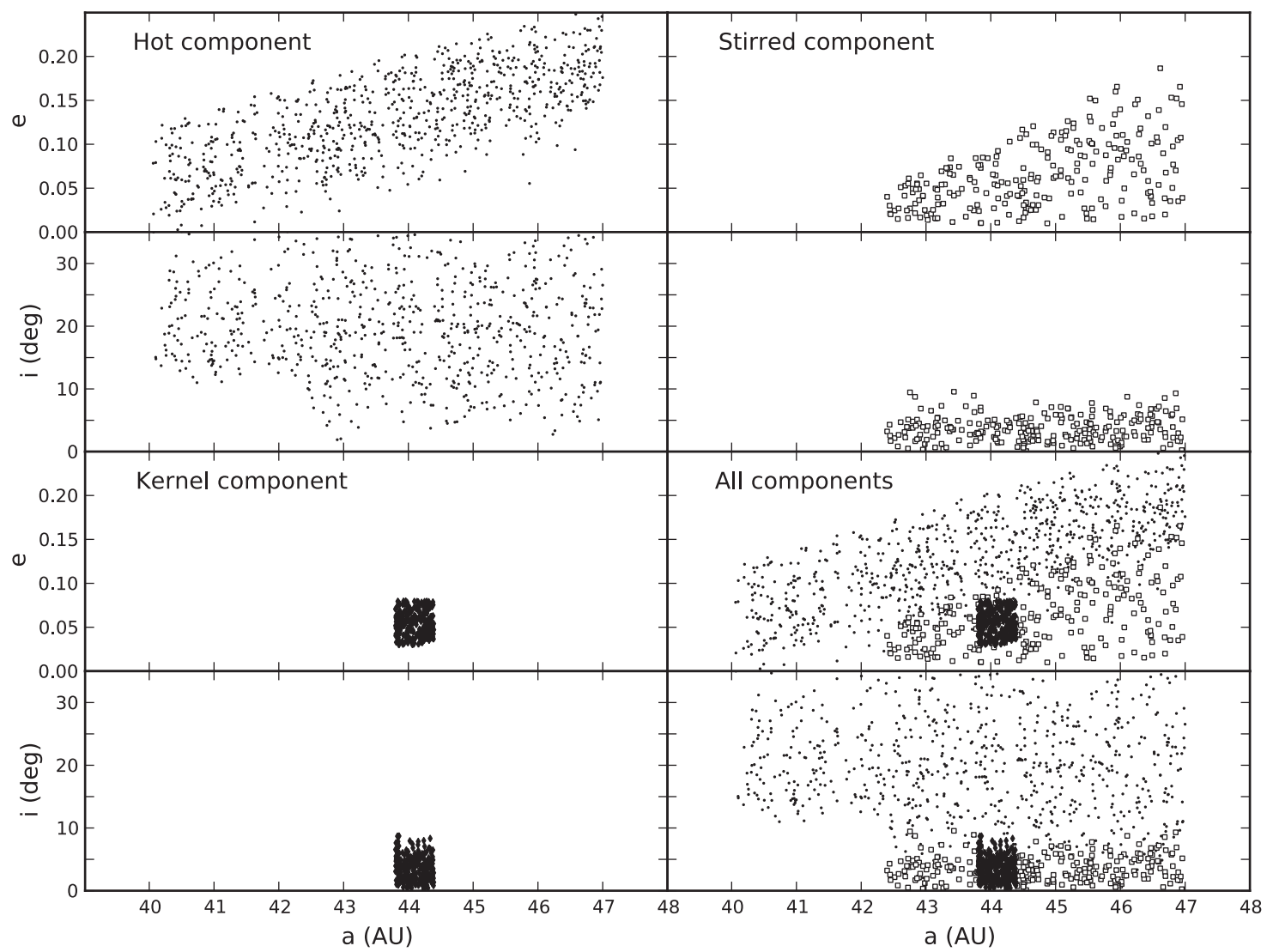

Figure 4. Three components of the CFEPS-L7 synthetic model for the main classical belt. The hole at low- $i$, low- $a$ in the hot component is introduced to represent the destabilizing action of the $v_{8}$ secular resonance.

that Chiang (2002) and Chiang et al. (2003b) posited (with rightful skepticism) as a possible collisional family. Although we share the concern that normally the relative speeds from a large parent-body breakup should be larger than this clump's observed dispersion, we find that regardless of interpretation, there is considerable observational support for a tightly confined structure in orbital element space near the location to which Chiang et al. pointed. Recent collisional modeling studies (e.g.,
Leinhardt et al. 2010) raise the possibility of grazing impacts forming low-speed families in the Kuiper Belt, motivated by the Haumea family (Brown et al. 2007). The large number of $D \geqslant 170 \mathrm{~km}$ (absolute magnitude ${ }^{14} H_{g} \leqslant 8$ ) objects in the kernel implies that the parent body would have been a dwarf

\footnotetext{
14 The $g$-band apparent magnitude of a TNO at heliocentric and geocentric
} distance of $1 \mathrm{AU}$ if viewed at $0^{\circ}$ phase angle. 
planet at least as large as Pluto, an unlikely possibility. The kernel thus appears to be the longest-recognized dynamical substructure in the classical Kuiper Belt, a structure which requires confinement in all of $a, e$, and $i$.

There may be other possible representations of the orbital distribution that are consistent with the CFEPS detections, with different boundaries or divisions of the phase space. We have found, however, the generic necessity of a threecomponent model cannot be avoided. The main characteristics of our model must be similar to reality, because a considerable amount of tuning was needed to achieve an acceptable model. From this three-component model, we can then provide robust measurements of the sizes of the sub-populations in the Kuiper Belt and generate a synthetic "de-biased" model of the orbital distribution of the main belt which can be used for various modeling purposes, such as collisional dust production (Stark \& Kuchner 2010).

\subsubsection{The Luminosity Function}

The absolute magnitude $H_{g}$ distribution can be represented by an exponential function

$$
N(\mathrm{H}) \propto 10^{\alpha \mathrm{H}}
$$

with "slope" $\alpha . H_{g}$ is converted into apparent magnitude $g$ by $g=H_{g}+2.5 \log \left(r^{2} \Delta^{2} \Phi(\mu)\right)$, where $\Delta$ is the geocentric and $r$ is the heliocentric distance, $\mu$ is the phase angle (Sun-TNOobserver) and $\Phi(\mu)$ is the phase function defined by Bowell et al. (1989). We find that two different values of $\alpha$, one for the hot and one for the cold distributions, are required by our observations. Allowing the stirred and kernel components to have differing values of $\alpha$ did not provide an improved match to the observations and is not required.

We have run a series of model cases using the orbital element distributions described previously while varying the luminosity function slopes for the hot component, $\alpha_{h}$, and for the cold (kernel + stirred) components $\alpha_{c}$. For each case, we varied the other orbit model parameters to find the best possible match between the cumulative distribution functions of the Survey Simulator observed Kuiper Belt and the L7 sample for each of the selected values of $\alpha_{h}$ and $\alpha_{c}$. In this way we determined the range of allowed power-law slopes for the limited range of TNO sizes, $7 \lesssim H_{g} \lesssim 8$, probed by our observations.

Our best-fit values are $\alpha_{c}=1.2_{-0.3}^{+0.2}$ and $\alpha_{h}=0.8_{-0.2}^{+0.3}$, with Figure 5 presenting the joint $95 \%$ confidence region for these slopes. A single value of $\alpha$ for all sub-components in our model is rejected at $>99 \%$ confidence. The $\alpha_{c}$ determined here is in good agreement with the range derived by Bernstein et al. (2004) for the low-inclination objects and somewhat steeper than that reported in Elliot et al. (2005) while our value for $\alpha_{h}$ overlaps the ranges proposed by both Bernstein et al. (2004) and Elliot et al. (2005) for what they call the excited population. Fraser et al. (2010) also found markedly different values for the slope of the cold component, 0.59-1.05, and the hot component, 0.14-0.56. While those slopes are consistent with Elliot et al. (2005) they are shallower than Bernstein et al. (2004) and our own estimates. The Fraser et al. (2010) results, however, probed smaller-size objects than our observations and the difference in slopes may be reflective of a change in size distribution around $H \sim 8.5$ where the CFEPS detections dwindle. Thus, in the limited-size ranges probed by these surveys, there appears to be reasonable agreement on the slope of luminosity function for these components of the Kuiper Belt with the hot and cold components exhibiting slopes that are significantly different.

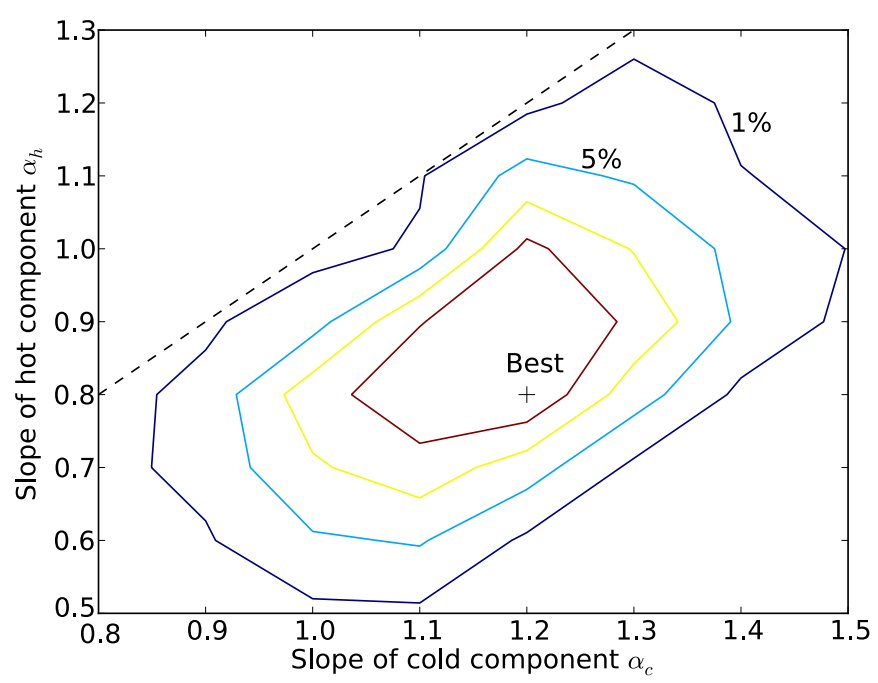

Figure 5. Contour plots of the "minimum probability" statistic for a range of main classical belt models. Each model has a different slope of the $H$ distribution for both the hot component $\left(\alpha_{h}\right)$ and the other components $\left(\alpha_{c}\right)$. Contour levels for $1 \%$ and $5 \%$ probabilities are shown. Acceptable models are interior to the $5 \%$ level curve. The dashed line indicates the locus with identical slopes for all components. The plus sign indicates the adopted model (which gave the best match).

(A color version of this figure is available in the online journal.)

The value of size distribution slopes reported here range from 0.8 to 1.2 and are considerably larger than the best-fit slopes discussed in many previous analyses that attempted to determine a global luminosity function for the Kuiper Belt. For example, Petit et al. (2008) reviewed estimates of $\alpha$ ranging from 0.5 to 0.8 for surveys that cover the range $H_{g} \simeq 5-10$. Fraser \& Kavelaars (2009) and Fuentes et al. (2009) demonstrated that a slope of $\sim \alpha=0.75$ is a decent representation of the "average" belt down to magnitude $\sim m_{r}=25$, but that there is a gradual flattening of the apparent luminosity-function slope at fainter magnitudes, continuing to a slope which may become extremely flat somewhere beyond $H>10$ according to the Bernstein et al. (2004) analysis of a deep Hubble Space Telescope search. The quest for a single "master" luminosity function, however, is misguided.

1. Because there are different slopes for the hot and cold main-belt components, the slope should be $\alpha \simeq 0.8$ at large sizes (where the hot component dominates) and become steeper (if looking in the ecliptic where the cold population is visible) when the depth of the survey results begins to probe the size range at which the cold-population surface density becomes comparable to the hot population.

2. The on-sky density of the (essentially non-resonant) cold population is essentially dependent only on the ecliptic latitude. The hot population's sky density varies with both latitude and longitude due to the fact that the resonant populations are hot. Thus, the $H$ magnitude at which the steeper cold component power law takes over will also depend on the latitude and longitude of the survey.

Interestingly, extrapolating from the $\sim 4000$ objects in the cold belt with $H_{g} \leqslant 8$ (see Section 5.1.3) to larger objects, one finds that there should be only $\sim 1$ TNO with $H_{g}<5$. This is consistent with the current census of large objects in the cold belt, which should be close to complete (Trujillo \& Brown 2003). Similarly, one would expect to have only $\sim 1$ TNO with $H_{g}<3.5$ in the non-resonant hot population, 
which again corresponds to our knowledge of the Kuiper Belt (Brown 2008). Currently, the MPC reports six objects with absolute magnitude $<3.5$ in the classical belt region as defined for our population estimate. Five of them are clearly part of the hot population, with inclinations between $20^{\circ}$ and $30^{\circ}$, and the last one is Quaoar with an intermediate inclination of $8^{\circ}$.

The realization that the hot component has a low- $i$ tail means that caution must be exercised because one simply cannot isolate the "cold" cosmogonic population with the commonly used $i<5^{\circ}$ cut. For example, in the ecliptic at bright (say roughly $m_{r} \sim 22$ ) magnitudes, the low- $i$ tail of the hot component can be numerically comparable to the sky density of "cold" objects. Thus, it is not possible to isolate the cold component at bright magnitudes based simply on orbital inclination.

\subsubsection{Acceptable Range for Main Parameters}

In this section we fix the slopes just determined, i.e., $\alpha_{h}=0.8$ and $\alpha_{c}=1.2$ and examine the range of model parameters allowed by the L7 detections. Due to the large number of orbital parameters to adjust and the time required by each survey simulation (10-50 minutes on the fastest available computers), we did not run an automated minimum-finding algorithm, but rather did a manual search on a multidimensional parameter grid.

Acceptable values (rejectable at less than 95\% confidence) for the inclination width (see Appendix A) of the hot component $\sigma_{h}$ range from $14^{\circ}$ to $29^{\circ}$. A hot-component width $\sigma_{h}=16^{\circ}$ is acceptable not just for the main-belt population but also reproduces the observed inner and outer classical populations (see Sections 5.2 and 5.3) and thus we adopt this value as the width of hot component.

The acceptable range for $\sigma_{c}$ is $2.3-3.5$, with a peak of the probability near 2.6 , which we adopt. Brown (2001) analyzed the MPC database at the time and concluded the existence of the cold component to the inclination distribution; with $\sigma_{c}=2.2_{-0.6}^{+0.2}$ deg ( $1 \sigma$ uncertainties $)$, consistent with our results. Elliot et al. (2005) in their initial analysis of the Deep Ecliptic Survey (DES) estimated a $1.94 \pm 0.19$ width for the cold component. Gulbis et al. (2010), however, recently re-analyzed the detections from the DES and found a $2.0_{-0.5}^{+0.6} \mathrm{deg}$ width $(1 \sigma$ uncertainties) for the cold component. Thus, the DES is also in reasonable agreement with our results, given the uncertainties. Brown \& Pan (2004) found a much narrower width of 1.3 (no uncertainty given) for the cold component, with respect to a locally determined Laplace plane for each semimajor axis. We have not repeated a similar analysis.

The L7 distribution contains an excess of intermediateinclination objects ( $i$ in range $6^{\circ}-10^{\circ}$ ) when compared to models with $\sigma_{h} \geqslant 16^{\circ}$ and $\sigma_{c}=2.2$. This "bump" in the cumulative inclination distribution can also be seen in the DES sample, Millis et al. (2002, Figure 13) and Elliot et al. (2005, Figure 17), between inclinations of $8^{\circ}$ and $10^{\circ}$. The Survey Simulator approach accounts for the distributions of all orbital elements simultaneously and thus the L7 model makes the inclination bump part of the cold component because the objects in this inclination range have $e$ and $a$ distributions that make them part of the cold component, hence increasing its width. Although it was possible to keep a cold width of 2.2 or lower by introducing a third inclination component the observations do not currently demand this increase in complexity.

The observed cumulative inclination distribution has two steep increases corresponding to the cold and hot components, both of which are steeper than for our model. This indicates that
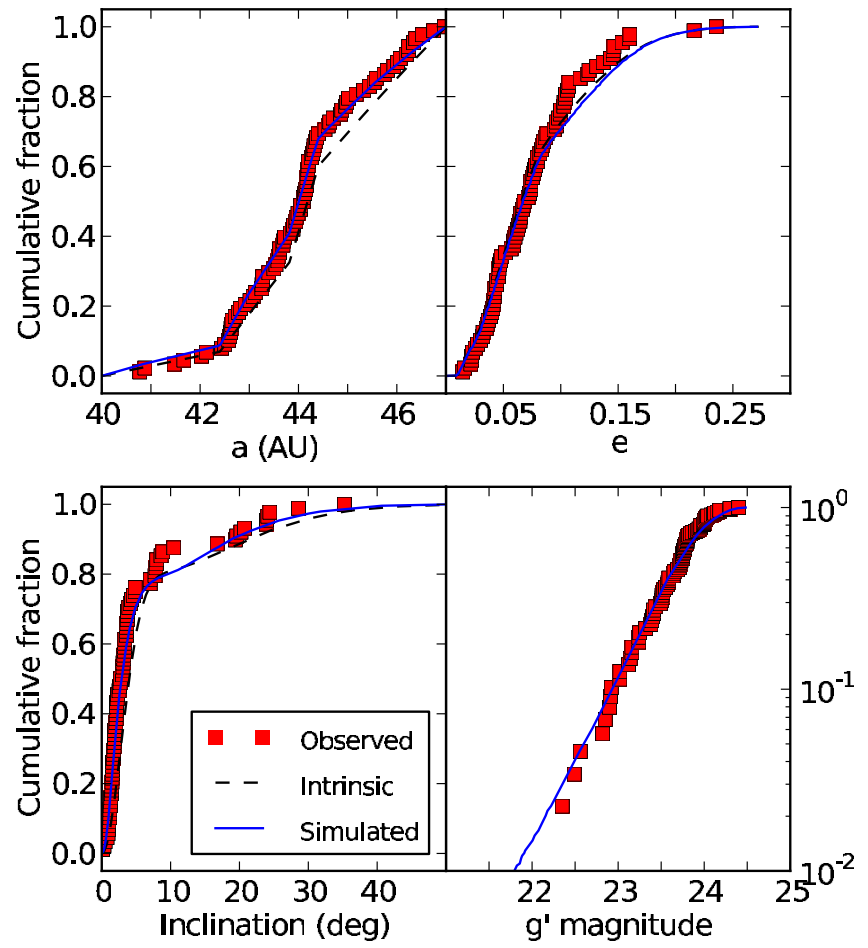

Figure 6. CFEPS+pre objects (red solid squares) compared to our mainbelt model's distribution in $a$ (upper left), $e$ (upper right), $i$ (lower left), and $g$ magnitude (lower right) distributions when the intrinsic (dashed line) are observed through the CFEPS Survey Simulator (resulting thin solid lines). The model used here is the one described in Section 5.1, with values of the parameters corresponding to our nominal case (see Section 5.1.2).

(A color version of this figure is available in the online journal.)

the actual differential distribution of each component is probably more confined than $\sin (i)$ times a Gaussian centered on zero. It is remarkable that the hot component of the main classical belt extends up to $35^{\circ}$ and stops abruptly. This limit is seen not only in the CFEPS, but also in the MPC databases (see Figures 2 and 3 ). We experimented with $\sin ^{2}(i)$ times a Gaussian centered on zero, but this did not result in a significant improvement to our fit. Note that Elliot et al. (2005) find that $\sin (i)$ times a Gaussian plus Lorenzian give their best fit to the classical belt inclination distribution. More recently, Gulbis et al. (2010) find that $\sin (i)$ times a Gaussian of width $\sim 7^{\circ}$ and centered around $\sim 20^{\circ}$ best fits what they call the "Scattered Object" inclination distribution. We did not test this functional form as this introduces an extra parameter, which is not demanded by the current sample. The Brown (2001) functional form may not be an exact representation of every sub-component's inclination distributions; we can, however, obtain an acceptable match to the CFEPS survey with this functional form.

The fraction of each component (hot versus cold inclination components) varies with the $H_{g}$-magnitude limit, due to their differing values of $\alpha$. We report here the acceptable range for the fractions of each sub-population at the $H_{g} \leqslant 8.0$ limit. We find that the fraction of the hot component, $f_{h}$, cannot exceed $62 \%$ and is at least $33 \%$, with a best match to the observations at $f_{h} \simeq 0.51$. This hot-component fraction and widths are close to the nominal L3 model from P1. We find that the fraction of the kernel $f_{k}$ has to be larger than 0.05 , but less than 0.30 at $95 \%$ confidence and adopt $f_{k}=0.11$. The fraction in the stirred is then $f_{s}=0.38$, when considering $H_{g}<8.0$.

Figure 6 presents the comparison of our nominal model with $a, e, i$, and $g$ apparent magnitude distributions. When biased 
by the CFEPS Survey Simulator, the L7-model reproduces the detections extremely well.

Our hot/cold population fractions differ from those reported in some other works, but details are important in the comparison. Brown (2001) report a hot fraction of $81 \%$. This fraction listed must be treated with the caution engendered by the realization that the MPC sample has a non-uniform $H$-magnitude limit, making interpretation of a fractional population (given the different luminosity functions) difficult. The Gulbis et al. (2010) estimate is even more difficult to compare, because the classification scheme used explicitly separates out many of the highest-inclination main-belt TNOs into portions of the "scattered" population (even though many of these TNOs are very decoupled from Neptune) and thus the relatively small "hot" width of $8_{-2}^{+3}$ has been forced down; a direct comparison of the relative populations is thus not possible. Trujillo et al. (2001) have an $H$-magnitude limit that is more uniform than the MPC sample but they mix together the various orbital classes when reporting the relative fraction of hot and cold component objects.

\subsubsection{Population Estimates}

The procedure in Section 4.3 of $\mathrm{P} 1$ was used to derive a population estimate for the main classical belt. Unlike much of the literature, which gives population estimates for objects larger than an estimated diameter, CFEPS gives population estimates for absolute magnitude smaller than a given value of $H_{g}$, and thus an unknown albedo is not introduced into the estimate. ${ }^{15}$ These estimates and their uncertainties are given assuming our orbital model. They would change if we were to change our parameterization. In particular, increasing the width of the inclination distributions "hides" more of the population far from the ecliptic. Alternately, decreasing the cold component's width to 1.3 requires changing the hot/cold fraction and results in a decrease of the total main-belt population by $20 \%$.

In principle, the very deepest blocks in our survey are sensitive to a limit of $H_{g} \simeq 9.5$ for a perihelion detection on the most eccentric orbits in our main-belt model. The Survey Simulator shows, however, that based on our model orbit distribution, the vast majority of our detections should have $H_{g} \leqslant 8.0$, consistent with our largest- $H$ classical-belter detection, $H_{g}=8.1$. Thus, our population estimate is given to the limit to which the survey has reasonable sensitivity:

$$
N_{\text {classical }}\left(H_{g} \leqslant 8.0\right)=\left(8000_{-1600}^{+1800}\right),
$$

where the uncertainties reflect a $95 \%$ confidence limit assuming the underlying orbital model and its parameter values are correct. Our measured value for $\alpha$ essentially is only for the range $H_{g}=7-8$ which dominate our detections.

The formula

$$
N\left(H_{g} \leqslant H_{1}\right)=10^{\alpha \times \Delta H} N\left(H_{g} \leqslant H_{0}\right),
$$

where $\Delta H=H_{1}-H_{0}$, allows one to scale population estimates of P1 to $H_{g}=8.0$, and also compare with other populations like the inner belt or the plutinos (which can come closer to Earth than the main classical belt). Here, care must be taken

\footnotetext{
15 More subtly, surveys at different latitudes and longitudes probe different average distances as they look into the trans-Neptunian region due to the different distance distributions of resonant and non-resonant populations; thus a given apparent magnitude depth actually probes at different average size limit. Stating a population limit to a stated $H$-magnitude limit is thus more meaningful.
}

Table 5

Model Dependent Population Estimates

\begin{tabular}{lcc}
\hline \hline & $N\left(H_{g} \leqslant 8\right)$ & $N(D \geqslant 100 \mathrm{~km})$ \\
\hline Population & Inner classical belt & \\
\hline All & $400_{-200}^{+400}$ & $3,000_{-2,000}^{+3,500}$ \\
Main classical belt & & \\
Hot & $4,100_{-800}^{+900}$ & $35,000_{-7,000}^{+8,000}$ \\
Stirred & $3,000_{-600}^{+700}$ & $75,000_{-15,000}^{+17,000}$ \\
Kernel & $900_{-200}^{+200}$ & $20,000_{-5,000}^{+5,000}$ \\
All & $8,000_{-2,000}^{+2,000}$ & $130,000_{-27,000}^{+30,000}$ \\
\hline & Outer/detached classical belt & \\
\hline All $(a>48)$ & $10,000_{-5,000}^{+7,000}$ & $80,000_{-40,000}^{+60,000}$ \\
\hline
\end{tabular}

Notes. Our model estimates are given for each sub-population within the Kuiper Belt. The uncertainties reflect $95 \%$ confidence intervals for the model-dependent population estimate. Values for $N(D>100 \mathrm{~km})$ are derived assuming an albedo of $p_{g}=0.05$, hence $H_{g}=9.16$. Remember that the relative importance of each population will vary with the upper $H_{g}$ limit.

to distinguish between the hot components and the others because they have different $H$-magnitude slopes, hence the extrapolation factor to any particular $H$-limit is different for each sub-component. Figure 7 shows a schematic representation of the fractional population sizes of all the dynamical classes measured in the L7 model. This figure demonstrates that one must be careful when comparing the relative sizes of various sub-populations whose size distributions are different because the relative populations will vary with the $H$-magnitude limit being considered.

Due to the lack of phase relations with Neptune and good statistics due to large numbers of main-belt detections in ecliptic surveys, the main classical belt population estimates should be the most certain in the literature of all the population estimates. Comparing the L7 main-belt estimate with the literature yields satisfactory agreement (details are given in Appendix C). Table 5 provides our current population estimates, after accounting for the size distribution scalings and using the same assumptions as in P1, i.e., an albedo of $p_{g}=0.05$, hence $H_{g}\left(D_{p}=100 \mathrm{~km}\right)=$ 9.16. Hahn \& Malhotra (2005) give an essentially identical estimate of 130,000 TNOs with 40.1 $<a<47.2 \mathrm{AU}$ and $D>100 \mathrm{~km}$ (with no error estimate), which is certainly within our $95 \%$ confidence region even with the small differences in albedo and phase-space boundaries used. If we use constant $\alpha$ values for the two components and extrapolate to $D>100 \mathrm{~km}$, we find the same population estimate as Hahn \& Malhotra (2005) and are a factor of a few higher than Trujillo et al. (2001) (see Appendix C).

Our current estimates agree with P1 when scaled to the $H_{g}<8$ limit where CFEPS is sensitive. Thus, it appears that the main-belt's population for $H_{g}<8$ is secure, where we have provided the first detailed breakdown of the hot and cold component's individual populations and detailed sub-structure.

\subsubsection{Discussion}

Some characteristics of the main classical belt that require explanation are the bimodal nature of the inclination distribution, the relative importance of the so-called hot component, and the marked sub-structures in $(a, e)$ space for the low-inclination objects.

Jewitt et al. (1998), Trujillo et al. (2001), Allen et al. (2001), Trujillo \& Brown (2001), and Kavelaars et al. (2008) reported 


$$
\mathrm{H}_{\vartheta}<8.0
$$

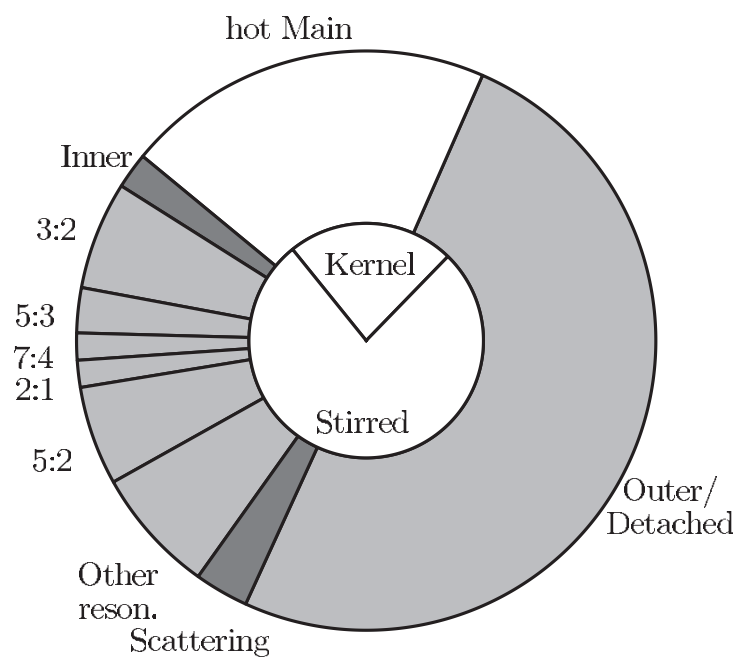

$$
\mathrm{H}_{9}<9.16(\mathrm{D}>100 \mathrm{~km})
$$

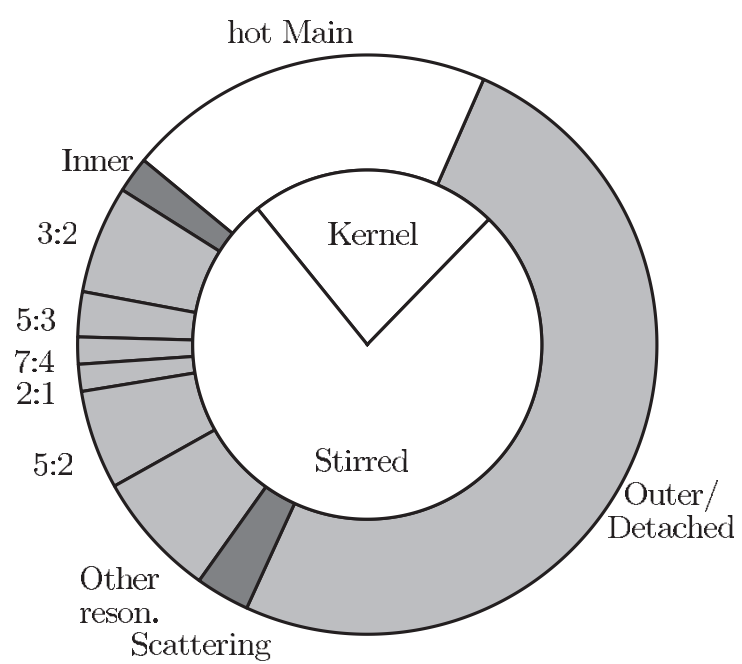

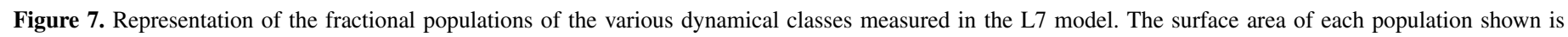

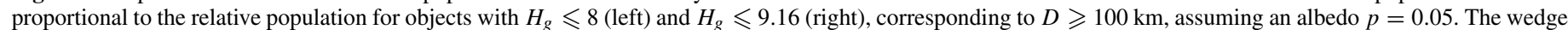

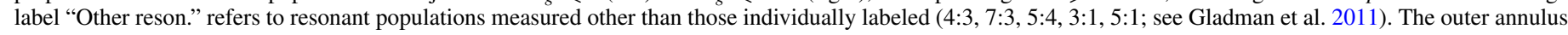

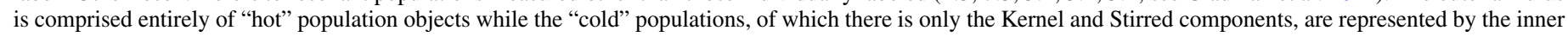
circle. The white area corresponds to the main belt.

the existence of an edge of the Kuiper Belt at 47-50 AU. Because the samples on which they based their estimate were heavily biased toward low-inclination objects, they were really detecting an edge of the cold component of the classical belt. In addition, Figure 14 of Trujillo et al. (2001), Figures 2 and 3 of Trujillo \& Brown (2001), and Figure 3 of Kavelaars et al. (2008) all show a marked peak at around $44 \mathrm{AU}$ followed by a very fast decrease in the number of objects past 44.5-45 AU, with perhaps a low density tail past $50 \mathrm{AU}$. The above papers vary in how sharp they consider the "cutoff" to be. In hindsight it is clear that what they were reporting as an edge is in fact due the presence of the low-inclination kernel and stirred components, which dominate the low-latitude detections and fall off quickly beyond 45 AU. As Kavelaars et al. (2008) point out, the peaked nature of the distribution is absent in the "hot" component and entirely absent from the "scattering disk" population. The stirred component's density is a rapidly decreasing function of semimajor axis that becomes very small by the time the $2: 1$ resonance is reached. This hints at a possible connection between the kernel, the stirred component, and the migration of the 2:1 to its current location; this outer edge appears only in the low-inclination component. We will show below that a scenario with the hot component continuous across the $2: 1$ resonance is in agreement with the data.

The L7 sample contains a cluster of six objects with large $e$ and $i$ just interior to the 2:1 MMR. Among these, only the one with $a<47 \mathrm{AU}$ (L4k17, $a=46.967$ ) was included in our analysis of models of the classical belt, the other five being in the region where the exact limit of the resonance is not easy to analytically define. This cluster could very well be a group of objects "dropped out" when the 2:1 MMR shrank at the end of Neptune's evolution (Section 7).

Gulbis et al. (2006) reported a difference between the B-R color of the "Core" and the "Halo," the former being redder than the latter, from photometric measurement they later acquired on the DES sample. Our orbital survey was also not designed to yield precision photometry, and the $g-i$ and $g-r$ colors that we can obtain from Table 7 are too uncertain to address this point.

In Section 8 we discuss some cosmogonic implications of these features, review how well the current models reproduce them, and propose future directions.

\subsection{The Inner Classical Belt}

The "inner" classical belt is the non-resonant and nonscattering population between Neptune and the 3:2 resonance. Paper P1 contained only two such TNOs, preventing us from deducing a detailed description of this region of the Kuiper Belt. There are six inner classical belt objects in the L7 sample (see Table 3), providing the opportunity to start constraining an orbital distribution. The phase space is cut by the $v_{8}$ secular resonance which eliminates almost all inner-belt TNOs with $7^{\circ}<i<20^{\circ}$ making the intrinsic inclination distribution difficult to interpret. If one uses a definition of "cold" belt as those objects with $i<5^{\circ}$ (e.g., Lykawka \& Mukai 2007), one concludes that a large fraction of the inner belt is cold. Such an analysis, however, neglects the bias toward detecting the lowest$i$ TNOs from the hot population in ecliptic surveys and the removal of moderate inclination objects via the $v_{8}$. Determining the intrinsic orbital distribution of the inner belt is precisely the sort of problem in which a simulator approach provides a clearer understanding.

\subsubsection{Parametric Model}

For the inner-belt population we utilized the same form of semimajor axis and perihelion distance distributions as for the hot component of the main classical belt (see Appendix A), changing the range of semimajor axis to be $37<a<39$ AU and fixing the size distributions for the hot and cold components to be the same as those found for the main-belt populations. We then attempted to find a model that included both a hot and a cold component using the same inclination widths and fractions as for the main belt, these models were rejected at $>95 \%$ confidence. 


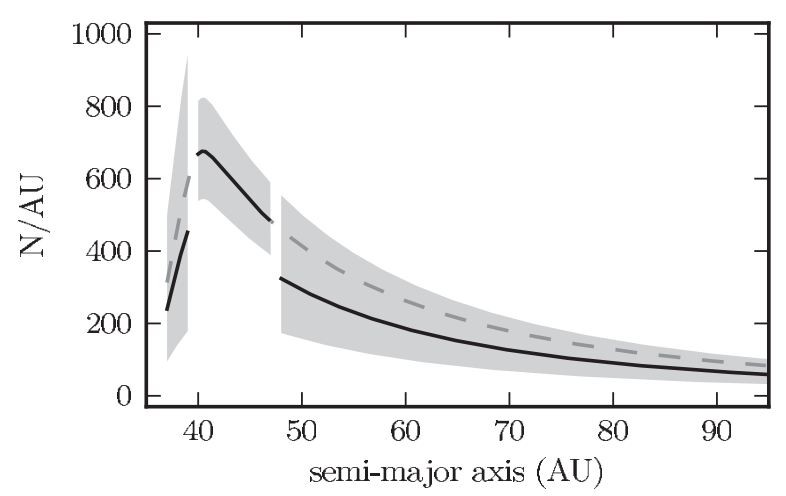

Figure 8. Linear number density (/AU) for three Kuiper Belt components: the inner belt $(a<39 \mathrm{AU})$, the hot main belt $(40<a<47)$, and the outer plus detached belts $(a>48 \mathrm{AU})$. Each region's total population is scaled to the number with $H_{g} \leqslant 8$, as determined by our model population estimates. The inner belt's population has been scaled up by a factor of 1.85 to account for the $v_{8}$ resonance (see footnote 16). The solid lines represent the model population determined independently for each zone while the gray dashed line indicates the smooth extension of the hot main-belt model to the semimajor axis range occupied by the inner belt and the outer+detached populations, where the inner-belt decay at lower $a$ occurs because of the rapidly shrinking stable $(a, q)$ phase-space volume available. A continuous primordial $a^{-2.5}$ hot population could, within uncertainties, account for all three populations. This suggests that these three Kuiper Belt components are a single dynamical population.

Using the same $(a, q)$ model but with a single-component inclination distribution width of $\sigma_{h}=16^{\circ}$, like the main-belt's hot component (cutting away $7^{\circ}<i<20^{\circ}$ orbits as they were proposed), provides a perfectly acceptable match to the L7 innerbelt detections. In fact, inclination widths of $5^{\circ}<\sigma_{h}<20.0$ were found to be acceptable. Even restricting one's attention only to the inner-belt TNOs with $i<7^{\circ}$ (inclinations below $v_{8}$ instability region) still requires an inclination distribution wider than the cold component of the main belt, indicating that the evidence against an inner-belt cold component comes from not just the largest- $i$ detections.

\subsubsection{Population Estimates}

Using a single component model with $\sigma_{h}=16^{\circ}$ and $\alpha=0.8$ we determine $N_{\text {inner }}\left(H_{g} \leqslant 8.0\right)=400_{-200}^{+400}$ (Table 5). This estimate is in good agreement with the L3-sample's estimate of $290_{-250}^{+690}$. As before, the uncertainties reflect $95 \%$ confidence limits given the intrinsic model distribution and does not reflect our uncertainty in the model. These random uncertainties are a factor of two, due to the small number of inner belt detection.

\subsubsection{Discussion}

Romanishin et al. (2010) compared photometric colors of inner-belt TNOs to other categories and found a good match between the inner belt and the high-inclination objects from the main belt, while a marked difference from the low inclination objects from the main belt, supporting the "hot-only" hypothesis. To attempt to duplicate the conclusion, we compared our photometric data for each population. Unfortunately, but also unsurprisingly, the quality of our photometric data is insufficient for such a comparison. The median uncertainty on our $g-i$ and $g-r$ colors is $\sim 0.25$, which is about five times more than for the Romanishin et al. (2010) data. We are thus unable to provide additional verification from our current photometric colors.

The successful use of the same orbital distribution for the inner-belt and the main-belt's hot component suggests that the entire inner belt may be the low- $a$ tail of the hot main belt. This would be a cosmogonically appealing unification of the sub-populations of the Kuiper Belt. If true, then (at least to order of magnitude) the TNO linear number density at the boundary (we chose 40 AU) extracted from each model should be comparable. Denoting $P\left(H_{g}<8.0\right)$ as the number of objects per AU with $H_{g}<8.0$, we find $P_{\text {inner }}\left(H_{g}<8.0,40 \mathrm{AU}\right)=270_{-100}^{+180} \mathrm{AU}^{-1}$. For the hot main belt, $P_{\text {main }}\left(H_{g}<8.0,40 \mathrm{AU}\right)=670_{-140}^{+160} \mathrm{AU}^{-1}$. At this interface, the hot main-belt number density is $\sim 3$ times that of the extrapolated inner belt. Given the very uncertain nature of these estimates and the fact that they are anywhere close leads us to postulate that the inner-belt and hot main TNOs were emplaced by a single cosmogonic process. In this hypothesis, the reduced inner-belt density would be due to the smaller volume of stable phase space in the inner belt region (because there is a smaller available stable range of $e$ ) as well as the significant range of inclinations from $7^{\circ}$ to $20^{\circ}$ destabilized by the $\nu_{8}$. Scaling the inner-belt population density, to account for this reduced inclination range, results in $P_{\text {inner }}\left(H_{g} \leqslant 8.0,40 \mathrm{AU}\right)=500_{-200}^{+300} \mathrm{AU}^{-1}$, consistent with the value from the main-belt estimate at the $2 \sigma$ level. Figure 8 presents the linear number density versus $a$ for the scaled inner belt ${ }^{16}$ compared to those of hot main-belt and outer+detached populations.

In this picture, the lack of a cold inner-belt component is significant. Assuming that the cold component originally existed in this region, the plausible mechanism for the cold component's destruction is the $v_{8}$ resonance sweeping out through the inner belt at some time, eliminating all low- $i$ TNOs. The nearby 3:2 MMR also lacks a cold component (Brown 2001; Kavelaars et al. 2008), which argues that if it swept slowly through the 36-39 AU region the cold component must have already been removed; otherwise, Hahn \& Malhotra (2005) show that the low$i$ objects should have been captured into the $3: 2$ and preserved (because the 3:2 shields its members from the effects of the $v_{8}$ ). One possible interpretation is that the 3:2 only obtained particles from a scattering hot population (as in the Levison et al. 2008 model) and ended with a large jump to its current location, but a reason for the lack of a cold population inside $39 \mathrm{AU}$ would need to be provided. Because the 3:2 location depends only on the semimajor axis of Neptune, one might expect that the 3:2 resonance's arrival at its current value would occur before the $v_{8}$ reaches its current location due to the latter's dependence on the orbital elements of multiple planets and the existence of other remaining mass in the system (Nagasawa \& Ida 2000).

\subsection{The Outer Edge of the Hot Belt}

We successfully construct a model of the non-resonant, nonscattering TNOs with semimajor axis beyond the 2:1 resonance by simply extending the $\mathrm{L} 7$ main-belt model out into this region. Using the classification system from Gladman et al. (2008), our current sample contains 3 outer-belt TNOs and 11 detached TNOs; the distinction between them is set by an arbitrary cut in eccentricity at $e=0.24$. For our current analysis, we group these two populations, under the hypothesis that they share a smoothly varying orbital distribution. ${ }^{17}$ In order to avoid problems with

\footnotetext{
16 The $7^{\circ}-20^{\circ}$ portion of a $\sin i \exp \left(-0.5 i^{2} /\left(16^{\circ}\right)\right.$ inclination distribution accounts for $46 \%$ of the $\sin i$-weighted phase space; to correct a population in the remaining phase space back to the original needs to be multiplied by $1 /(1-0.46)=1.85$.

17 Although it remains to be seen if the very large inclination objects like Buffy (Allen et al. 2006) or Drac (Gladman et al. 2009) are part of such a distribution.
} 
the exact border of the 2:1, we start our modeling at $a=48 \mathrm{AU}$; this eliminated 1 detached TNO, reducing our sample to 13 .

The outer/detached objects share the same $(q, i)$ distributions as the hot main classical belt. This suggests that again (as for the inner belt) the outer population may be a smooth extension of the main-belt hot component. To model the outer/detached TNOs, we thus use the same prescription as for the hot main classical belt, with $\alpha=0.8$ and an $a$ range from 48 AU to a value $a_{\max }$, with density varying as $a^{-\beta}$, with $\beta=2.5$. We tried varying the exponent $\beta$ of the $a$ distribution. For shallow distributions, i.e., $\beta \leqslant 1.5$, the model is rejected when $a_{\max }$ exceeds $\sim 100 \mathrm{AU}$, because it creates too many simulated detections close to $a_{\max }$. The range $2.0 \leqslant \beta \leqslant 3.0$ produces acceptable models with no constraint on $a_{\max }$. Models with larger values of $\beta$ exhibit a very steep decrease of number density at large $a$ and fail to produce enough detections with $a>60$ AU. We thus adopt $\beta=2.5$, as for the main classical belt. The number of objects needed to reproduce our 13 outer/detached detections is insensitive to our choice of $a_{\max }$ due to the strong detection biases. Hence we formulate our population estimate for a population with no outer edge, finding a population beyond $48 \mathrm{AU}$ of $N_{\text {outer } / \text { detached }}\left(H_{g} \leqslant 8.0\right)=10,000_{-5,000}^{+7,000}$ (see Table 5). Of these, only a small number $N_{\text {outer }}\left(H_{g} \leqslant 8.0\right)=500_{-250}^{+350}$ have $e<0.24$, thus belonging to the outer belt defined by Gladman et al. (2008).

As for our analysis of the inner belt, we computed the number density of TNOs per unit $a$ at a main/outer interface at $47 \mathrm{AU}, P_{\text {outer } / \text { detached }}\left(H_{g} \leqslant 8.0,47 \mathrm{AU}\right)=340_{-150}^{+230} \mathrm{AU}^{-1}$ and compare it to the value from the outer edge of the mainbelt $P_{\text {main,outer }}\left(H_{g} \leqslant 8.0\right)=490_{-100}^{+110} \mathrm{AU}^{-1}$. Hence the TNO number density per unit $a$ in the outer/detached belt is the same as that of the hot main belt, within uncertainties. There was absolutely no coupling in the debiasing procedure of these two TNO populations; this matching result was not tuned in any way. Figure 8 demonstrates that an initially uniform semimajor axis distribution for all three of these Kuiper Belt sub-components as a single dynamical population is a plausible scenario.

Given the number of papers discussing a noticeable edge to the distribution (Jewitt et al. 1998; Allen et al. 2001; Trujillo \& Brown 2001) this continuity may be surprising. Realize that the continuity is in the hot component, which our analysis indicates is actually present throughout the region from Neptune to at least several hundred AU. This population has a pericenter distribution with very few $q$ 's above 40 AU, and may very well have been emplaced as a sort of fossilized scattered disk (Gladman et al. 2002) as illustrated in Morbidelli \& Levison (2004). This same process, however, does not emplace the kernel and stirred components which dominate the main-belt region for $H_{g}>8.0$ nor produce the dramatic falloff beyond $45 \mathrm{AU}$ in these cold populations.

\section{THE SCATTERING DISK}

If the Centaurs and then JFCs do indeed come from one of the Kuiper Belt's sub-populations, then their penultimate metastable source will be the set of TNOs currently scattering off Neptune, as defined by Morbidelli et al. (2004) and Gladman et al. (2008). Hence we wish to give a population estimate for this "actively scattering" population. Unfortunately the region occupied by the scattering objects is not a simply connected region definable by a simple parameter-space cut; they are intimately mixed with stable resonant and non-resonant objects and providing a full dynamical model of this region is well beyond the scope of the current manuscript. Here we examine available models of the scattering disk using the CFEPS Survey Simulator to provide an order of magnitude population estimate for this important transient population.

The definition of the scattering population has evolved over the last 15 years. A cosmogonic perspective is easily adopted by workers doing numerical simulations. In such simulations the "scattered" disk is taken to be comprised of TNOs that currently do not have encounters with Neptune but were delivered onto those orbits via an encounter. Morbidelli et al. (2004) quantified this definition by requiring that scattered TNO needs to have it semimajor axis change by more than $1.5 \mathrm{AU}$ over the life of the solar system. In this process, knowledge of orbital history is required for classification and, clearly, this information is not available for a given real TNO. More problematically, if the Levison et al. (2008) model is correct then the entire Kuiper Belt would qualify as having scattered off Neptune, making the term scattered disk object a meaningless distinction. Gladman et al. (2008) proposed a practical definition for classification based on the orbit of known objects at the current epoch, in which the "scattering objects" are those currently (in the next $10 \mathrm{Myr}$ ) undergoing scattering encounters with Neptune in a forward simulation. In the current manuscript we consider two definitions of the scattering disk, one based on a parameterized region of phase space and one based on numerical modeling of a particular scattering process, to derive an estimate of the scattering population.

Both Trujillo et al. (2000) and Hahn \& Malhotra (2005) give population estimates of the scattered Kuiper Belt, but based on different definitions of this population. The former called the scattered Kuiper Belt the region of phase space $50 \mathrm{AU} \leqslant a \leqslant 200 \mathrm{AU}$ and $34 \mathrm{AU} \leqslant q \leqslant 36 \mathrm{AU}$. Based on their detection of four objects with preliminary orbits in this region, they provide a population estimate of 18,000-50,000 objects ( $1 \sigma$ range) with $D>100 \mathrm{~km}$, assuming an $H_{g}$ distribution slope of 0.8 . Using CFEPS and the same orbit and $H_{g}$ distributions as Trujillo et al. (2000) we estimate the population in that region of the phase space to be $2100-17,500$ objects $(95 \%$ confidence range), about a factor of four less than Trujillo et al. (2000)'s estimate. This estimate is based on scaling the Survey Simulator's detections to match all the L7 detections in this range of $q$. Awkwardly, none of the L7 detections with orbits in this $q$ range are actually members of the scattering class, thus this estimate is more correctly an estimate of some restricted portion of the Detached population. Two of the objects (1999 $\mathrm{CV}_{118}$ and $1999 \mathrm{CF}_{119}$ ) used by Trujillo et al. (2000) for their population estimate were later found to not have orbits in the region they called the scattered disk, hence their population estimate of this region should be divided by 2 , making it more compatible with our estimate. ${ }^{18}$ The choice of the $q=34-36$ AU region was motivated by the candidate "scattering" orbits known to Trujillo et al. (2000), intending this to be a source region for the Centaurs and JFCs, as postulated by Duncan \& Levison (1997). However, the majority of the known TNOs in that phase space cut are not currently interacting with Neptune and are on resonant or detached orbits (Gladman et al. 2008). A simple phase-space cut is not appropriate for the scattering disk population.

To obtain an order-of-magnitude population estimate via a dynamical model, we used the result of numerical integrations by Gladman \& Chan (2006). This model attempted to produce

\footnotetext{
18 The other two sources (1999 $\mathrm{TL}_{66}$ and $1999 \mathrm{CY}_{118}$ ) are found to be on scattering obits (Gladman et al. 2008).
} 
Table 6

Scattering Disk Population Estimates

\begin{tabular}{lcc}
\hline \hline Population & $N\left(H_{g} \leqslant 10\right)$ & $N(D>100 \mathrm{~km})$ \\
\hline Scattering disk & $25,000_{-15,000}^{+20,000}$ & $5000_{-3,000}^{+5,000}$ \\
\hline
\end{tabular}

the detached population via secular interaction with rogue planets, where the additional planet persists for the first $200 \mathrm{Myr}$ of the simulation. Gladman \& Chan (2006) find that the scattering particles in their simulations that survive to the end of the a 4 Gyr integration largely forget their initial state. To obtain a scattering disk model, we selected the orbital elements of actively scattering test particles during the last $500 \mathrm{Myr}$ of one $4.5 \mathrm{Gyr}$ integration. We then slightly smeared the orbital elements and applied an $H$-magnitude distribution with slope $\alpha=0.8$. We found a reasonable match between the orbital elements of the L7 scattering sub-population and our input scattering model, as observed by the Survey Simulator, although the inclination distribution was somewhat too cold, yielding a confidence level of only $8 \%$. The apparent magnitude distribution was rejected at more than $99 \%$ whatever the slope of the $H_{g}$ distribution we used; it is plausible that this rejection is due to a change in luminosity-function slope in the size range probed by our observations, as the faintest absolute magnitude of our detections is $H_{g}=10$ because scattering TNOs include many $q<30$ AU members. Three of the CFEPS active scatterers were inside $30 \mathrm{AU}$ at the time of detection. The match between the orbital model and the observations allows us to be reasonably confident that our population estimate is good to a factor of 10 and we do not feel that this order of magnitude estimate warrants further tuning until a larger sample of scattering objects is in hand. While there is clearly future room to better test models, we give here the first published estimate of the active scattering population. The results are given in Table 6 for $H_{g}<10$ and for diameter $D>100 \mathrm{~km}\left(H_{g}<9.16\right.$ assuming an albedo of $\left.p_{g}=0.05\right)$. The quoted factor-of-three uncertainty accounts only for the Poisson variation.

We estimate an actively scattering population that is about $2 \%-3 \%$ that of the sum of the classical belts. Interpretation of this number is problematic. A very large actively scattering population would require that the current disk could not be the steady state intermediary between the Centaurs and a longerlived source in the trans-Neptunian region, in which case the currently actively scattering population is more likely to be the long-lived tail of a roughly 100 times more populous primordial population (Duncan \& Levison 1997). For the 2\%-3\% figure, the active scatterers could conceivably be now dominated by objects that have left the resonant, detached, or classical populations in the last Gyr.

\section{TESTING COSMOGONIC KUIPER BELT MODELS}

The CFEPS-L7 model is an empirical parametric model that properly reproduces the observed orbital distribution of the Kuiper Belt, once passed through our survey simulator. The purpose of this parametric model is to provide absolutely calibrated population estimates of the various sub-populations of the Kuiper Belt. The model also exhibits important features of the intrinsic Kuiper Belt that a cosmogonic model should reproduce. For example, one needs to produce a cluster of objects at low inclination and low eccentricity near $44 \mathrm{AU}$, that we call the kernel. There is also a low- $i$ component extending from the outer edge of the $v_{8}$ secular resonance at $42.4 \mathrm{AU}$ out to the 2:1 MMR with Neptune. Finally, there is a hot component with a confined $q$ range that extends in semimajor axis from the inner belt at $\sim 35$ AU out to several hundred AU with a decreasing surface density. The synthetic L7 model is also useful for observational modeling of our Kuiper Belt, with Stark \& Kuchner (2010) as an example for the outer solar system dust distribution based on the L3 model.

The ability to provide a detailed quantitative comparison with a cosmogonic model is, however, the true power of the CFEPS survey. This is done by passing a proposed model of the current Kuiper Belt distribution through the CFEPS survey simulator and then comparing this detection-biased model with the real CFEPS detections. Through this procedure one can choose between models in a statistically robust way. Both the CFEPS L7 synthetic model and the CFEPS survey simulator are available from the project Web site http://www.cfeps.net.

Several models have been proposed to explain the dynamical structure of the Kuiper Belt (Malhotra 1993; Ida et al. 2000; Hahn \& Malhotra 2005; Levison et al. 2008, to name a few). Since the primary purpose of CFEPS was to validate or refute cosmogonic models, we present an example of this process. Because we had available both an orbital element distribution and a resonance-occupation analysis (H. F. Levison 2010, private communication), we have chosen to use Run B of Levison et al. (2008) as an example of how one uses the CFEPS Survey Simulator to compare a model to the observed Kuiper Belt. The simulation in question (motivated by the Nice model of the re-arrangement of the outer solar system) has already some known problems pointed out by its authors, but the model's intriguing aspects make it a good example of the comparison process.

All the fictitious TNOs in the Run B model were dynamically classified following the Gladman et al. (2008) procedure (C. Van Laerhoven 2010, private communication). The final planetary configuration in the Nice model was intentionally made different from that of the solar system to avoid the $v_{8}$ secular resonance inadvertently sweeping through and destroying the belt and thus has many objects in the $40<a<42.4$ AU range at low inclination, where the real $v_{8}$ resonance would eliminate them. To avoid this complication, we restricted the comparison to the range $42.4<a<47 \mathrm{AU}$, yielding 128 non-resonant model TNOs from run $\mathrm{B}$.

We use the following procedure to generate the large number of TNOs required as input to the Survey Simulator. First we select a model object at random, and vary the orbital elements uniformly by $\pm 0.2 \mathrm{AU}$ in both $a$ and $q$ and \pm 0.5 in inclination and then randomize the elements $\Omega, \omega$, and $M$. There was no size distribution given for the model objects, so the $H_{g}$ magnitude of each object was drawn from an exponential distribution (see the equation in Section 5.1.1). Given the orbital elements and $H_{g}$ magnitude, we then use our Survey Simulator to determine if the model object would have been detected by the CFEPSL7 observations. We repeat the procedure until we have a set number of simulated detections and then compare the $a, e, i, q$, and $r$ one-dimensional cumulative distributions of the simulated detections to those of the $\mathrm{L} 7$ sample, using the AD test.

For the $H_{g}$ distribution, we tried single slopes of $0.6 \leqslant \alpha \leqslant$ 1.3 and also a model with $\alpha=1.2$ for the low- $i$ and $\alpha=0.8$ for the high-inclination objects, as in our favored model. All the models for the $H_{g}$ distribution produced acceptable matches to the apparent magnitude distribution, but had no effect on the orbital element distributions, so we do not show the magnitude distribution. 

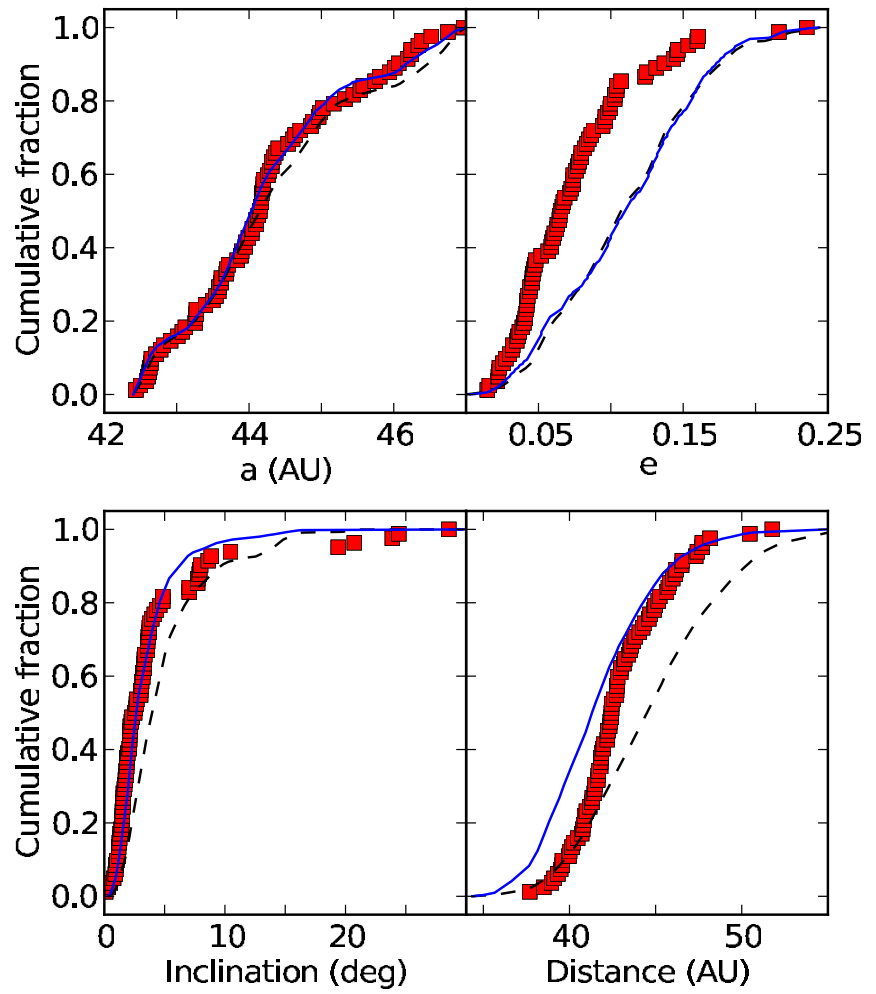

Figure 9. CFEPS+pre objects (red solid squares) compared to the Nice model's distribution in $a$ (upper left), $e$ (upper right), $i$ (lower left), and $r$ (lower right) distributions when the intrinsic (black dashed line) distribution is biased via the CFEPS Survey Simulator (resulting blue solid lines). This shown case corresponds to a single-slope $\alpha=1.1 \mathrm{H}_{\mathrm{g}}$ distribution.

(A color version of this figure is available in the online journal.)

Figure 9 compares the distribution of $a, e, i$, and $r$ of the L7 sample and simulated detections from the model. There is remarkably good agreement for the $a$ distribution. Although the Nice model does not exhibit a clustering around $44 \mathrm{AU}$ as strong as the L7 sample, the difference between the two distribution is not statistically significant.

On the other hand, the model's $e$ distribution is too excited compared to the observed one, as already noted by Levison et al. (2008). This then results in detection distances that are overly dominated by small-distance detections. For both cumulative distributions, the $\mathrm{AD}$ test says that the hypothesis that the observed objects could be drawn from the model can be rejected at $>99.9 \%$ confidence.

The model's $i$ distribution is not a good match either, again as already noted by Levison et al. (2008); the AD test rejects the $i$ distribution at more than $99.9 \%$ confidence. This is mostly because the L7 distribution has two components, while the Run $\mathrm{B}$ input model gives an essentially unimodal distribution. The simulated detections from the model appear roughly consistent up to $i \simeq 4^{\circ}$, but there is a lack of high- $i$ TNOs, to which the AD test is sensitive. Run B lacks the hot component that peaks between $15^{\circ}$ and $20^{\circ}$ and extends past $30^{\circ}$. Looking only at the $i<6^{\circ}$ region, here too the AD test rejects the model at more than $99.9 \%$ confidence. Most of the Run B classical belt comes from the low- $i$ outer part of the planetesimal disk, which acquires inclinations similar to that of the inner initial disk, with a width $6^{\circ}$. The true cold component, on the contrary, has a width certainly less than $3^{\circ}$. We conclude that one needs a strongly bi-modal input population to the Nice model in order to produce the desired bi-modal inclination distribution we see in the real Kuiper Belt. More worrying is the claim by Levison et al. (2008) that increasing the width of the initial population produced the same final inclination distribution, meaning there is a missing ingredient in this model to explain the dynamical structure of the Kuiper Belt. Levison et al. (2008) mention another simulation, Run E, which generated too many hot objects compared to the cold population; so there may be an intermediate parameter set that could match the observations.

\section{CONCLUSIONS}

This paper's modeling concerns the non-resonant Kuiper Belt, although the L7 release lists all detections from the CFEPS survey fields from 2002 to 2007 for the sake of completeness. Due to the complexity of the modeling required because of the phase relations with Neptune, the resonant populations are presented in a separate paper (Gladman et al. 2011). We find that the debiased orbital and $H$-magnitude distributions show that there is considerable sub-structure in the main Kuiper Belt. We quantitatively measured the size of the various sub-populations, created an empirical model of these sub-populations in the L7 synthetic model, and provide an algorithm (the CFEPS survey simulator) to quantitatively compare cosmogonic models to the intrinsic Kuiper Belt. Here we summarize the results and offer a synthesis and interpretation.

A plausible hypothesis is that the hot population permeates the entire Kuiper Belt region from $30 \mathrm{AU}$ up to at least $200 \mathrm{AU}$, albeit with a projected surface density (onto the invariable plane) that decreases with semimajor axis. Even the resonant populations are consistent with the idea that the entire hot component is a vestigial "fossilized" scattered disk from an epoch when TNOs with perihelia up to $\sim 40$ AU were being weakly scattered by a massive object at the inner edge of the Kuiper Belt (whether this object was Neptune or something else is unclear from the present data). The inclination distribution of this hot population can be represented by $\sin (i)$ times a Gaussian of width $\sim 16^{\circ}$. Note, however that, due to the strong bias against detection of large- $i$ objects in an ecliptic survey, our current sample does not provide a strong constraint on the width or the functional form of the hot component. A scenario in which the inner belt, hot main belt, outer belt, and detached populations, along with the resonant populations were all emplaced simultaneously from a population scattered outward during the final stages of planet formation, with a single size distribution, initial inclination distribution, color distribution, and binary fraction, is an attractive hypothesis. The plausibly continuous initial number density across the inner/ main and main/outer boundary (see Figure 8) supports this idea.

The Kuiper Belt's (surviving) "cold" population is entirely confined between semimajor axes of 42.4 AU and the 2:1 resonance with Neptune, and its inclination distribution (measured relative to the $\mathrm{J} 2000$ ecliptic) is adequately represented via $\sin (i)$ times a Gaussian of width 2.6 , with an acceptable range from 2.3 up to 3.5 . There are indeed $i<5^{\circ}$ TNOs in the low- $i$ tail of the hot population all over the Kuiper Belt and even in the $42.4<a<47$ AU region, so an inclination cut does not provide a clean separation between the hot and cold components of the main belt. In the current belt we claim that all $i<5^{\circ}$ TNOs with semimajor axis outside the above range are the hotcomponent objects that happen to have lower inclinations. The cold population exhibits a particularly strong grouping in band of about $1 \mathrm{AU} a$ thickness, centered at $44 \mathrm{AU}$ (which we call the kernel). The linear number density (\#/AU) of "cold" belt objects increases from the inner edge at $42.4 \mathrm{AU}$ up to a maximum 
at $24.4 \mathrm{AU}$, all with rather low eccentricities. Past 44.4 AU, the linear number density drops noticeably, and classical TNOs tend to have higher eccentricities; the CFEPS-L7 model uses a "stirred" population that covers the 42.4-47 AU range with a single parameterization. We favor the idea that this cold component is primordial (the objects formed at roughly their current heliocentric distances), although this is not required.

The primordial distance range of the cold population is difficult to constrain. The inner boundary at $a=42.4$ AU may have been eroded via scattering by massive bodies and resonance migration; an important condition is that any sequence of events cannot allow either the inner belt or the mean-motion and secular resonances that probably migrated through it to have preserved a cold component today. The coincidence of the stirred population's outer edge with the 2:1 resonance suggests to us that the kernel marks the original outer edge and that the larger- $a$ cold objects have either (1) been dragged out of the $a<44.4$ AU region via trapping and then drop-off in the 2:1 as it went past (in the fashion studied by Hahn \& Malhotra 2005) or (2) due to weak scattering out of the $40<a<44.4$ AU region. Perhaps the edge of the original cold population around $45 \mathrm{AU}$ may be explained by the global evolution of solid matter in turbulent protoplanetary disks (Stepinski \& Valageas 1996, 1997), although an even-more extreme density contrast may be needed at $\sim 30$ AU to prevent Neptune's continued migration outward (Gomes et al. 2004). Sharp drops in surface density are commonly observed in protoplanetary disks at about this 30-50 AU scale (Johnstone et al. 1998; Mann \& Williams 2009, 2010).

There is an issue with a primordial origin of the cold population at this location. The on-ecliptic mass density of this population is extremely low and it would be difficult to form multi-hundred km TNOs in a low surface density environment. This may not be impossible due to recent work on forming planetesimals big (Morbidelli et al. 2009a; Youdin 2011), which can be favored by external photoevaporation (Throop \& Bally 2005) and may be supported by the fact that it appears that there are simply no cold objects larger than $H \sim 5$; all the larger objects are in the other populations which may come from closer to the Sun where the mass density was higher.

The kernel around $44 \mathrm{AU}$ is an intriguing feature. A collisional family explanation would eliminate the idea that the $44.4 \mathrm{AU}$ edge is a primordial edge, but would instead simply be where the very low velocity dispersion breakup occurred. This velocity dispersion is even lower than for the putative Haumea collisional family (Brown et al. 2007). An additional puzzle is the unclear significance that the kernel is bounded between the $7: 4$ and the 9:5 MMRs. One possible, very ad hoc, explanation would be that the 2:1 MMR started its migration interior to $43.5 \mathrm{AU}$ while being wide (due to a large Neptune eccentricity), and then had a stochastic jump by a few tenth of AU while near $44 \mathrm{AU}$, leaving behind a pile of objects that we see as the kernel today.

The hot population poses other strong constraints. Models by Gomes (2003), Hahn \& Malhotra (2005), and Levison et al. (2008) all succeed in creating a hot population that has a similar radial extent to what is currently observed, but have varying success in matching the inclination distribution. When slowly migrating Neptune over long distances (>8 AU) into an initially cold disk, Gomes (2003) and Hahn \& Malhotra (2005) generated a reasonable TNO fraction with inclinations up to $35^{\circ}$. When migrating Neptune over a shorter distance (Gomes 2003), or in a hot disk (Hahn \& Malhotra 2005), the fraction of high- $i$ TNOs is noticeably reduced, while still reaching the same maximum i. Levison et al. (2008), on the contrary, migrate Neptune over a short distance (2-3 AU) into a warm scattered disk (with $\langle i\rangle=6^{\circ}$ ) and essentially maintain the input inclination distribution. They report that increasing the initial $i$ distribution resulted in the same final population, which lacks TNOs with $i>30^{\circ}$ and which we have confirmed is colder than the actual belt. These facts appear to indicates that Neptune had to slowly migrate over a long distance in a cold disk in order to obtain the observed inclination distribution of the hot population. However, Morbidelli et al. (2009b) showed that a long and slow migration of Neptune, coupled with a similar migration of the other giant planets, does not correctly reproduce the secular architecture of the solar system, in particular the amplitudes of the eigenmodes characterizing the current secular evolution of the eccentricities of Jupiter and Saturn. They conclude that only the Nice model can reproduce the current dynamics of the inner solar system and the giant planets. Unfortunately this scenario does not produce Kuiper Belt components with orbital properties that agree with the $\mathrm{L} 7$ orbit catalog.

The idea that the hot population originated from a planetesimal population scattered outward by Neptune, whose resonant and largest- $q$ members are preserved, is extremely attractive. Thus much of Levison et al.'s (2008) general scenario has many pleasing aspects and one is tempted to think of the hot population as the transplanted population, even if our results show that the inclination distribution is a stumbling block. Contrary to some statements (e.g., Fraser et al. 2010), we find that the Nice model is not good at producing the hot population's inclination distribution, but surprisingly produces rather well the cold population's fine structure in the semimajor axis distribution. The large- $i$ TNOs which do appear could instead be interpreted as coming from the "evader" mechanism of Gomes (2003). In this conception the Fraser et al. (2010) finding, that the luminosity distribution of the Jovian Trojans is more similar to the cold than hot TNO populations, makes perfect sense in a scenario in which the injection of bodies into the Jovian Trojan region occurs from the same source region as the implantation of the Kuiper Belt's cold population. In the Nice model this seems unlikely because Jovian Trojan capture occurs just after the Jupiter-Saturn mutual 1:2 resonance crossing (Morbidelli et al. 2005) and involves small bodies closer to the planets than the cold outer disk that is the main source of the cold Kuiper Belt. If so, the hot component cannot be generated from the Nice model's inclined inner disk, as this would have the same size distribution as the Jovian Trojans. One needs another source for the hot population, one that is not too perturbed by the initial instability in Neptune's motion. A final caveat concerns the existence of wide binaries in the cold belt; Parker \& Kavelaars (2010) showed that the Neptune scattering occurring in the Nice model would disrupt nearly all such wide binaries, thus requiring a more gentle mechanism to move the cold belt to its current location if that population did not form in situ.

Our current understanding of the trans-Neptunian region is not likely to advance rapidly for timescales of order a decade unless new surveys begin to efficiently probe TNOs that were rare in the ecliptic surveys. The most likely approach that would result in an advance are moderate-depth (24th magnitude) wide-field surveys (many hundreds of square degrees) at higher ecliptic latitudes, or deeper (25th magnitude) surveys covering $\sim 100 \mathrm{deg}^{2}$ targeting regions of sky that attempt to isolate cosmogonically interesting sub-populations. We hope that CFEPS will serve as a standard for the need for well-characterized discovery and tracking. The Large Synoptic 
Survey Telescope (LSST) (Ivezic et al. 2008) should certainly firm up the main-belt dynamical sub-structure along with the color and size distributions for those components.

This research was supported by funding from the Natural Sciences and Engineering Research Council of Canada, the Canadian Foundation for Innovation, the National Research Council of Canada, and NASA Planetary Astronomy Program NNG04GI29G. This project could not have been a success without the dedicated staff of the Canada-France-Hawaii telescope as well as the assistance of the skilled telescope operators at KPNO and Mount Palomar.

We dedicate this paper to the memory of Brian G. Marsden, for his devotion to orbital determination and passionate encouragement to observational planetary astronomers.

In addition to CFHT (MegaPrime) this work was made possible thanks to access to facilities listed in Table 2.

\section{Facility: CFHT (MegaPrime)}

\section{APPENDIX A}

In this appendix, we give details of the algorithm used to generate the CFEPS-L7 model of the main classical belt.

The main classical belt objects are constrained in what is essential three-dimensional phase space due to the (confirmed a posteriori) fact that the mean anomaly and longitudes of ascending node and perihelion are all uniformly distributed in the intrinsic population. Thus the L7 model consists of three subpopulations constrained by three orbital-element distributions to determine for each sub-population.

The inclination distribution of each subcomponent is well represented by a probability distribution proportional to $\sin (i)$ times a Gaussian $\exp \left[i^{2} /\left(2 \sigma^{2}\right)\right]$, where past results indicate a "cold"-component width of $\sim 2.5$ and a "hot"-component width of $\sim 15^{\circ}$ (Brown 2001; Kavelaars et al. 2008).

The hot component occupies the semimajor axis range from 40.0 to $47.0 \mathrm{AU}$ and is defined by

1. an $a$ distribution with a probability density function (PDF) proportional to $a^{-5 / 2}$, corresponding to a surface density proportional to $a^{-7 / 2}$;

2. an inclination distribution proportional to $\sin (i) \times$ $\exp \left[i^{2} /\left(2 \sigma_{h}^{2}\right)\right]$, with width $\sigma_{h}=16^{\circ}$;

3. we eliminate objects from the region unstable due to the $v_{8}$ secular resonance: $a<42.4 \mathrm{AU}$ and $i<12^{\circ}$;

4. a perihelion distance $q$ distribution that is mostly uniform between 35 and $40 \mathrm{AU}$, with soft shoulders at both ends extending over $\sim 1 \mathrm{AU}$; the PDF is proportional to $1 /([1+$ $\exp ((35-q) / 0.5)][1+\exp ((q-40) / 0.5)]) ;$ any object with $q<34 \mathrm{AU}$ is rejected;

5. finally, we reject objects with $q<38-0.2 i$ (deg) to account for weaker stability of low- $q$ orbits at low inclination.

We have found that the exact form of the truncation at low perihelion distance is unimportant, as long as the limiting value of $q$ is a decreasing function of the inclination; this is justified dynamically as low-inclination orbits cannot have $q<38$ AU and remain stable (Duncan et al. 1995).

The stirred component covers only the range of stable semimajor axis at low inclinations:

1. an $a$ distribution with PDF proportional to $a^{-5 / 2}$ between 42.4 (limit of the $v_{8}$ resonance) and $47 \mathrm{AU}$;
2. a uniform $e$ distribution between 0.01 and a maximum value depending on the semimajor axis, $e_{\max }=0.04+(a-42) \times$ 0.032 , to reproduce the structure seen in Figures 2 and 3;

3. randomly keep objects with probability $1 /(1+\exp [(e-$ $0.6+19.2 / a) / 0.01])$, which corresponds to a soft cut at $q=38+0.4 *(a-47)$

4. an inclination distribution proportional to $\sin (i)$ times a Gaussian of width $\sigma_{c}=2.6$;

5. again, we reject objects with $q<38-0.2 \times i(\mathrm{deg})$ as for the hot component.

Finally, the kernel provides the group of objects with low inclination in the middle of the main classical belt as seen in Figure 2:

1. a uniform $a$ distribution between 43.8 and $44.4 \mathrm{AU}$;

2. a uniform $e$ distribution between 0.03 and 0.08 ;

3 . an inclination distribution proportional to $\sin (i)$ times a Gaussian of width $\sigma_{c}=2.6$, identical to the stirred population.

For all components, the remaining orbital elements (longitude of node, argument of perihelion, and mean anomaly) are drawn at random uniformly between $0^{\circ}$ and $360^{\circ}$. All elements are generated in the invariable plane reference frame (inclination $1^{\circ}$ $35^{\prime} 13^{\prime \prime}$. 86 with respect to J2000 ecliptic plane with direction of ascending node at $107^{\circ} 36^{\prime} 30^{\prime}$. 8 ). In particular, we state widths of the inclination distribution with respect to the invariable plane. Elliot et al. (2005), Brown \& Pan (2004), and Gulbis et al. (2010) studied the distribution of inclinations with respect to their selfdetermined Kuiper Belt plane, which differ from the invariable plane.

To evaluate the acceptability of each model we evaluate our parameterization in distinct portions of phase space.

1. $i \geqslant 10 \mathrm{deg}$,

2. $i<10 \mathrm{deg}$,

3. $a>44.4 \mathrm{AU}$,

4. $a \leqslant 44.4 \mathrm{AU}$, and

5. the entire main-belt region.

We computed the probability of the AD or KKS statistics in each region separately and consider the minimum on all element distributions and all sub-regions when determining if a particular parameterization is rejected.

The variable parameters are the $i$-width of the hot component $\sigma_{h}$, the cold component's $i$ width $\sigma_{c}$, the $H$-magnitude distribution of these two components (with slopes $\alpha_{h}, \alpha_{c}$ ), the hot population's fraction of the main-belt $f_{h}$, and the kernel fraction $f_{k}$, with the stirred component forming the remainder: $f_{s}=1-f_{h}-f_{k}$.

The CFEPS-L7 model has the following known weaknesses.

1. Resonant orbits will be generated by chance in the mainbelt region (especially for the 5:3, 7:4, and 9:5 resonances).

2. The $v_{8}$ resonance cut is done in osculating, rather than proper, orbital elements space and thus some L7 model objects near the resonance will be unstable.

3. There are four tiny semimajor axis gaps in our model: small regions $(\sim 0.3 \mathrm{AU}$ in $a)$ on both sides of the $3: 2$ and $2: 1$ resonances.

\section{APPENDIX B}

The CFEPS project is built on the observations acquired as the "Very Wide" component of the CFHT Legacy Survey 
(CFHTLS-VW). All discovery imaging data are publicly available from the Canadian Astronomy Data Centre $\left(\mathrm{CADC}^{19}\right)$. These images were acquired using the CFHT Queue Service Observing (QSO) system. For each field observed on a photometric night the CFHT QSO provides calibrated images using their ELIXIR processing software (Magnier \& Cuillandre 2004). Our photometry below is reported in the Sloan system (Fukugita et al. 1996) with the calibrations contained in the header of each image as provided by ELIXIR. Color corrections were computed using the average color for Kuiper Belt objects $(g-r) \sim 0.7$. Differential aperture photometry was determined for each of our detected objects observed on photometric nights and these fluxes are reported in Table 7. All CFEPS discovery observations were acquired in photometric conditions in a relatively narrow range of seeing conditions due to queue-mode acquisition. The photometry below supercedes information that may be in the MPC's observational database.

\section{APPENDIX C}

Comparing previously published population estimates of the main classical belt, either as a whole or for the various components, with our present values must ensure that the same limiting $H_{g}$ magnitude and the same region of the phase space are adopted. The main difference between P1 and the present L7 model is the higher $q$ cutoff that was applied to the P1 sample. Restricting our current sample to the same region of phase space as was modeled in P1 gives very similar population estimates for the main belt. Second, P1's cold component was restricted in extend to $a \leqslant 45 \mathrm{AU}$ and hence had a smaller population than in our current model, and conversely, the hot population was slightly overestimated compared to our current value, for the region of phase space. Lastly, because we use widely different $H$-magnitude slopes, the population estimates should be compatible for the detected $H_{g}=7-8$ range, but diverge for smaller TNOs (larger H). Scaling P1 to the $H_{g}=8$ limit, we find

$$
N_{\mathrm{P} 1}\left(H_{g} \leqslant 8.0\right)=4400_{-1100}^{+1800},
$$

while restricting our current model to the same phase space gives

$$
N_{\mathrm{All}}\left(H_{g} \leqslant 8.0\right)=5800_{-1200}^{+1300},
$$

in reasonable agreement.

The latest independent population estimate of the main classical belt was done by Trujillo et al. (2001), who estimate $38,000_{-2700}^{+5400}$ objects bigger than $D=100 \mathrm{~km}$, with uncertainties being $3 \sigma$ confidence. This number is more than three times smaller than our $H_{g}=9.16$ estimate. Can the two numbers be reconciled?

First, one must match the size ranges of the population being estimated. Trujillo et al. used a red albedo $p_{R}=0.04$ and a solar red magnitude of -27.1 . In this case a TNO of $D=100 \mathrm{~km}$ has an absolute magnitude $\mathrm{H}_{R}=8.8$. Assuming the same $g-R=$ 0.8 color as we used in Kavelaars et al. (2009), this corresponds to $H_{g}=9.6$. When looking at Figure 9 of Trujillo et al. (2001) one clearly sees that either the assumption of an exponential luminosity function breaks at around $m_{R} \sim 24$ or the debiasing is incorrect faintward of that value. In particular, the lack of

\footnotetext{
19 http://www.cadc.hia.nrc.gc.ca
}

Table 7

\begin{tabular}{|c|c|c|c|c|c|c|c|c|c|}
\hline Object & $g$ & $\sigma_{g}$ & $N_{g}$ & $r$ & $\sigma_{r}$ & $N_{r}$ & $i$ & $\sigma_{i}$ & $N_{i}$ \\
\hline L3f01 & 23.66 & 0.41 & 4 & 23.12 & 0.15 & 3 & $\ldots$ & $\ldots$ & .. \\
\hline L3f04PD & 22.74 & 0.33 & 4 & $\ldots$ & $\ldots$ &.. & $\ldots$ & $\ldots$ & .. \\
\hline L3h01 & 23.83 & 0.27 & 4 & 23.02 & 0.11 & 3 & $\ldots$ & $\ldots$ & .. \\
\hline L3h04 & 24.32 & 0.17 & 4 & 23.82 & 0.33 & 7 & $\ldots$ & $\ldots$ & .. \\
\hline L3h05 & 24.36 & 0.07 & 2 & 23.56 & 0.30 & 7 & $\ldots$ & $\ldots$ & .. \\
\hline L3h08 & $\ldots$ & $\ldots$ & .. & 23.15 & 0.75 & 7 & $\ldots$ & $\ldots$ & .. \\
\hline L3h09 & 22.73 & 0.04 & 4 & 22.29 & 0.23 & 10 & $\ldots$ & $\ldots$ & .. \\
\hline L3h11 & 23.44 & 0.20 & 7 & 23.13 & 0.10 & 3 & $\ldots$ & $\ldots$ & .. \\
\hline L3h13 & 23.73 & 0.10 & 4 & 23.29 & 0.27 & 7 & $\ldots$ & $\ldots$ & .. \\
\hline L3h14 & 23.27 & 0.15 & 3 & 22.81 & 1.42 & 8 & $\ldots$ & $\ldots$ & .. \\
\hline L3h18 & 23.42 & 0.09 & 3 & 22.53 & 0.17 & 8 & $\ldots$ & $\ldots$ & .. \\
\hline L3h19 & $\ldots$ & $\ldots$ &.. & 23.67 & 0.26 & 9 & $\ldots$ & $\ldots$ & .. \\
\hline L3h20 & $\ldots$ & $\ldots$ &.. & 23.15 & 0.30 & 7 & $\ldots$ & $\ldots$ & .. \\
\hline L3q01 & 23.89 & 0.21 & 3 & 22.96 & 0.17 & 3 & 22.76 & 0.36 & 3 \\
\hline L3q02PD & 23.50 & 0.10 & 3 & 22.49 & 0.07 & 4 & 22.23 & 0.02 & 3 \\
\hline L3q03 & 23.19 & 0.17 & 4 & 22.36 & 0.25 & 4 & 22.37 & 0.00 & 1 \\
\hline L3q04PD & 24.15 & 0.43 & 4 & 23.31 & 0.14 & 4 & 23.10 & 0.16 & 3 \\
\hline L3q06PD & 23.58 & 0.30 & 4 & $\ldots$ & $\ldots$ &.. & $\ldots$ & $\ldots$ & .. \\
\hline L3q08PD & 23.67 & 0.18 & 3 & $\ldots$ & $\ldots$ &.. & $\ldots$ & $\ldots$ & .. \\
\hline L3q09PD & 23.50 & 0.24 & 4 & $\ldots$ & $\ldots$ &.. & $\ldots$ & $\ldots$ & .. \\
\hline L3s01 & 23.54 & 0.12 & 6 & $\ldots$ & $\ldots$ &.. & 22.54 & 0.12 & 2 \\
\hline $\mathrm{L} 3 \mathrm{~s} 02$ & 23.81 & 0.28 & 6 & 23.40 & 0.23 & 4 & 23.18 & 0.11 & 2 \\
\hline $\mathrm{L} 3 \mathrm{~s} 03$ & 22.90 & 0.20 & 5 & $\ldots$ & $\ldots$ &.. & 22.65 & 0.23 & 3 \\
\hline $\mathrm{L} 3 \mathrm{~s} 05$ & 23.67 & 0.30 & 5 & $\ldots$ & $\ldots$ &.. & 22.88 & 0.13 & 2 \\
\hline L3s06 & 22.82 & 0.03 & 5 & & $\ldots$ &.. & 21.89 & 0.07 & 3 \\
\hline L3w01 & 22.89 & 0.61 & 5 & $\ldots$ & $\ldots$ &.. & $\ldots$ & $\ldots$ & .. \\
\hline L3w02 & 23.56 & 0.11 & 4 & 22.80 & 0.08 & 4 & 22.54 & 0.07 & 3 \\
\hline L3w03 & 23.76 & 0.07 & 5 & 22.50 & 0.08 & 4 & $\ldots$ & $\ldots$ & .. \\
\hline L3w04 & 22.44 & 0.03 & 5 & 21.65 & 0.04 & 4 & 21.53 & 0.02 & 3 \\
\hline L3w05 & 24.20 & 0.31 & 4 & 23.70 & 0.17 & 3 & 23.77 & 0.07 & 3 \\
\hline L3w06 & 23.65 & 0.25 & 4 & 23.13 & 0.19 & 4 & $\ldots$ & $\ldots$ & .. \\
\hline L3w07 & 22.95 & 0.09 & 5 & $\ldots$ & $\ldots$ &.. & 22.46 & 0.09 & 3 \\
\hline L3w08 & 23.96 & 0.13 & 4 & $\ldots$ & $\ldots$ &.. & 22.79 & 0.14 & 3 \\
\hline L3w09 & 23.53 & 0.10 & 3 & 22.72 & 0.15 & 4 & $\ldots$ & $\ldots$ & .. \\
\hline L3w10 & 23.95 & 0.20 & 5 & 23.04 & 0.13 & 4 & 22.00 & 0.63 & 3 \\
\hline L3w11 & 24.03 & 0.12 & 4 & 23.49 & 0.15 & 4 & 23.34 & 0.13 & 3 \\
\hline L3y01 & 24.04 & 0.26 & 4 & 22.62 & 0.55 & 3 & $\ldots$ & $\ldots$ & .. \\
\hline L3y02 & 23.38 & 0.09 & 6 & 22.69 & 0.19 & 4 & $\ldots$ & $\ldots$ & .. \\
\hline L3y03 & 23.41 & 0.09 & 4 & 22.79 & 0.11 & 4 & $\ldots$ & $\ldots$ & .. \\
\hline L3y05 & 23.89 & 0.03 & 4 & 22.99 & 0.12 & 3 & $\ldots$ & $\ldots$ & .. \\
\hline L3y06 & 23.37 & 0.18 & 3 & $\ldots$ & $\ldots$ &.. & $\ldots$ & $\ldots$ & .. \\
\hline L3y07 & 23.42 & 0.09 & 4 & 22.87 & 0.23 & 5 & $\ldots$ & $\ldots$ & .. \\
\hline L3y09 & 23.65 & 0.17 & 4 & 23.01 & 0.14 & 5 & $\ldots$ & $\ldots$ & .. \\
\hline L3y 11 & 23.82 & 0.32 & 4 & 23.51 & 0.21 & 4 & $\ldots$ & $\ldots$ & .. \\
\hline L3y12PD & 21.73 & 0.03 & 4 & 20.81 & 0.04 & 4 & $\ldots$ & $\ldots$ & .. \\
\hline L3y14PD & 23.68 & 0.19 & 4 & 22.73 & 0.10 & 3 & $\ldots$ & $\ldots$ & .. \\
\hline $13 \mathrm{f} 05$ & 23.71 & 0.18 & 3 & $\ldots$ & $\ldots$ &.. & $\ldots$ & $\ldots$ & .. \\
\hline 13h10 & $\ldots$ & $\ldots$ &.. & 23.03 & 0.28 & 7 & $\ldots$ & $\ldots$ & .. \\
\hline $13 \mathrm{~h} 15$ & $\ldots$ & $\ldots$ &.. & 23.74 & 0.27 & 7 & $\ldots$ & $\ldots$ & .. \\
\hline $13 \mathrm{~h} 16$ & $\ldots$ & &.. & 23.53 & 0.64 & 7 & $\ldots$ & $\ldots$ & .. \\
\hline $13 q 05$ & 23.68 & 0.16 & 4 & $\ldots$ & $\ldots$ &.. & $\ldots$ & $\ldots$ & .. \\
\hline 13q07 & 24.19 & 0.21 & 7 & $\ldots$ & $\ldots$ & .. & $\ldots$ & $\ldots$ & .. \\
\hline $13 w 14$ & 23.98 & 0.20 & 3 & $\ldots$ & $\ldots$ &.. & $\ldots$ & $\ldots$ & .. \\
\hline $13 w 19$ & 24.00 & 0.11 & 3 & & $\ldots$ &.. & $\ldots$ & $\ldots$ & .. \\
\hline U3f02 & 24.07 & 0.21 & 4 & 23.41 & 0.31 & 3 & $\ldots$ & $\ldots$ & .. \\
\hline U3h06 & $\ldots$ & $\ldots$ & .. & 23.96 & 0.35 & 7 & $\ldots$ & $\ldots$ & .. \\
\hline $\mathrm{U} 3 \mathrm{~s} 04$ & 24.10 & 0.45 & 6 & $\ldots$ & $\ldots$ &.. & $\ldots$ & $\ldots$ & .. \\
\hline U3w13 & 24.39 & 0.21 & 4 & 23.96 & 0.21 & 3 & $\ldots$ & $\ldots$ & .. \\
\hline U3w16 & 24.07 & 0.14 & 4 & $\ldots$ & $\ldots$ &.. & 23.14 & 0.25 & 3 \\
\hline U3w17 & 24.45 & 0.09 & 4 & $\ldots$ & $\ldots$ &.. & 23.27 & 0.15 & 3 \\
\hline U3y04 & 24.25 & 0.13 & 4 & 22.57 & 2.16 & 4 & $\ldots$ & $\ldots$ & .. \\
\hline U3y08 & 24.25 & 0.13 & 4 & 23.43 & 0.25 & 3 & $\ldots$ & $\ldots$ & .. \\
\hline U3y16 & 23.93 & 0.37 & 4 & 23.45 & 0.16 & 3 & $\ldots$ & $\ldots$ & .. \\
\hline u3h02 & $\ldots$ & 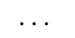 & .. & 23.96 & 0.25 & 4 & $\ldots$ & $\ldots$ & .. \\
\hline u3h03 & $\ldots$ & $\ldots$ &.. & 24.01 & 0.09 & 3 & $\ldots$ & $\ldots$ & .. \\
\hline u3h07 & $\ldots$ & $\ldots$ &.. & 24.35 & 0.43 & 4 & $\cdots$ & $\ldots$ & .. \\
\hline
\end{tabular}

Object Fluxes 
Table 7

(Continued)

\begin{tabular}{|c|c|c|c|c|c|c|c|c|c|}
\hline Object & $g$ & $\sigma_{g}$ & $N_{g}$ & $r$ & $\sigma_{r}$ & $N_{r}$ & $i$ & $\sigma_{i}$ & $N_{i}$ \\
\hline u3h12 & .. & $\cdots$ &.. & 23.79 & 0.23 & 7 & $\cdots$ &. & .. \\
\hline u3h17 & $\ldots$ & . &.. & 24.17 & 0.33 & 4 & 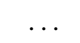 & $\ldots$ & .. \\
\hline u3w12 & 24.43 & 0.15 & 3 & $\ldots$ & $\ldots$ & .. & 23.48 & 0.26 & 3 \\
\hline u3w15 & 24.07 & 0.21 & 3 & $\ldots$ & $\ldots$ & .. & $\ldots$ & $\ldots$ & .. \\
\hline u3w18 & 24.49 & 0.19 & 3 & $\ldots$ & $\ldots$ & .. & $\ldots$ & $\ldots$ & .. \\
\hline u3y10 & 24.00 & 0.09 & 3 & $\ldots$ & $\ldots$ & .. & $\ldots$ & $\ldots$ & .. \\
\hline u3y 13 & 24.28 & 0.16 & 3 & $\ldots$ & $\ldots$ & .. & $\ldots$ & $\ldots$ & .. \\
\hline L4h01PD & 23.77 & 0.40 & 3 & $\ldots$ & $\ldots$ & .. & $\ldots$ & $\ldots$ & .. \\
\hline L4h02PD & 23.50 & 0.18 & 3 & $\ldots$ & $\ldots$ & .. & $\ldots$ & $\ldots$ & .. \\
\hline L4h05PD & 23.96 & 0.16 & 3 & 23.48 & 0.31 & 4 & $\ldots$ & $\ldots$ & .. \\
\hline L4h06 & 23.83 & 0.12 & 3 & 22.77 & 0.75 & 2 & $\ldots$ & $\ldots$ & .. \\
\hline L4h07 & 23.70 & 0.08 & 3 & 23.35 & 0.37 & 6 & 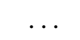 & $\ldots$ & .. \\
\hline L4h08 & 23.01 & 0.03 & 4 & 22.72 & 0.13 & 3 & 22.41 & 0.14 & 3 \\
\hline L4h09PD & 21.34 & 0.18 & 4 & $\ldots$ & $\ldots$ & .. & $\ldots$ & $\ldots$ & .. \\
\hline L4h10PD & 22.96 & 0.09 & 4 & $\ldots$ & $\ldots$ & .. & 22.62 & 0.16 & 3 \\
\hline L4h11 & 22.98 & 0.09 & 4 & 22.52 & 0.10 & 4 & 22.08 & 0.09 & 3 \\
\hline L4h12 & 24.11 & 0.34 & 4 & 22.92 & 0.35 & 4 & 22.60 & 0.01 & 3 \\
\hline L4h13 & 23.64 & 0.23 & 3 & 23.34 & 0.13 & 3 & 22.53 & 0.15 & 2 \\
\hline L4h14 & 23.68 & 0.30 & 4 & 23.65 & 0.31 & 3 & $\ldots$ & $\ldots$ & .. \\
\hline L4h15 & 24.05 & 0.05 & 3 & 23.74 & 0.63 & 7 & & $\ldots$ & .. \\
\hline L4h16 & 24.04 & 0.45 & 3 & 23.38 & 0.25 & 3 & $\ldots$ & $\ldots$ & .. \\
\hline L4h18 & 23.34 & 0.38 & 4 & 23.54 & 1.00 & 3 & 22.01 & 0.25 & 3 \\
\hline L4h21 & 23.75 & 0.05 & 3 & 23.43 & 0.32 & 3 & 23.00 & 0.12 & 3 \\
\hline L4j01 & 23.81 & 0.18 & 5 & 23.46 & 0.40 & 7 & & $\ldots$ & .. \\
\hline L4j02 & 23.35 & 0.09 & 5 & 22.59 & 0.13 & 7 & 22.37 & 0.12 & 3 \\
\hline L4j03 & 23.87 & 0.13 & 5 & 23.06 & 0.18 & 7 & 22.91 & 0.15 & 3 \\
\hline L4j05 & 23.55 & 0.10 & 6 & 22.66 & 0.12 & 6 & 22.46 & 0.14 & 3 \\
\hline L4j06PD & 22.13 & 0.04 & 4 & 21.70 & 0.03 & 4 & 21.74 & 0.04 & 2 \\
\hline L4j07 & 22.96 & 0.19 & 5 & 22.10 & 0.09 & 3 & 21.91 & 0.04 & 2 \\
\hline L4j08 & 23.51 & 0.16 & 5 & 22.79 & 0.20 & 7 & 22.43 & 0.32 & 3 \\
\hline L4j10 & 23.75 & 0.21 & 3 & 23.06 & 0.12 & 2 & 22.82 & 0.06 & 3 \\
\hline L4j11 & 23.55 & 0.32 & 8 & 23.04 & 0.30 & 5 & 22.77 & 0.26 & 3 \\
\hline L4j12 & 23.61 & 0.08 & 4 & 23.14 & 0.11 & 3 & $\ldots$ & $\ldots$ & .. \\
\hline L4k01 & 24.01 & 0.12 & 3 & 23.11 & 0.17 & 2 & 23.03 & 0.11 & 3 \\
\hline $\mathrm{L} 4 \mathrm{k} 02$ & 23.14 & 0.20 & 4 & 22.59 & 0.04 & 2 & 22.18 & 0.15 & 2 \\
\hline L4k03 & 23.54 & 0.28 & 4 & 22.97 & 0.34 & 9 & 22.25 & 0.05 & 3 \\
\hline L4k04 & 24.16 & 0.15 & 4 & 23.22 & 0.03 & 3 & 22.96 & 0.12 & 3 \\
\hline L4k09 & 23.69 & 0.22 & 4 & 22.53 & 0.29 & 3 & 22.34 & 0.19 & 4 \\
\hline L4k10 & 24.43 & 0.23 & 4 & 23.44 & 0.28 & 4 & 23.19 & 0.15 & 3 \\
\hline L4k11 & 23.32 & 0.15 & 4 & 22.99 & 0.10 & 3 & 22.58 & 0.07 & 2 \\
\hline $\mathrm{L} 4 \mathrm{k} 12$ & 23.20 & 0.20 & 4 & 22.72 & 0.11 & 4 & & $\ldots$ & .. \\
\hline L4k13 & 23.99 & 0.16 & 3 & 23.15 & 0.12 & 3 & 23.03 & 0.17 & 3 \\
\hline L4k14 & 24.08 & 0.14 & 4 & 23.32 & 0.19 & 4 & 22.93 & 0.95 & 3 \\
\hline L4k15PD & 23.22 & 0.07 & 4 & 22.48 & 0.17 & 3 & 22.12 & 0.02 & 2 \\
\hline L4k16 & 23.99 & 0.15 & 4 & 22.93 & 1.27 & 5 & 23.32 & 0.12 & 2 \\
\hline L4k17 & 23.07 & 0.17 & 4 & 22.55 & 0.12 & 5 & 22.33 & 0.05 & 3 \\
\hline L4k18 & 23.61 & 0.09 & 4 & 23.08 & 0.34 & 3 & $\ldots$ & $\ldots$ & .. \\
\hline L4k19 & 23.66 & 0.29 & 4 & 23.40 & 0.17 & 6 & $\ldots$ & $\ldots$ & .. \\
\hline L4k20 & 23.80 & 0.27 & 4 & 23.19 & 0.29 & 3 & 22.47 & 0.46 & 2 \\
\hline $\mathrm{L} 4 \mathrm{~m} 01$ & 23.83 & 0.14 & 5 & $\ldots$ & $\ldots$ & .. & $\ldots$ & $\ldots$ & .. \\
\hline $\mathrm{L} 4 \mathrm{~m} 02$ & 23.43 & 0.25 & 8 & 22.72 & 0.10 & 3 & & $\ldots$ & .. \\
\hline $\mathrm{L} 4 \mathrm{~m} 03$ & 23.57 & 0.39 & 5 & 23.33 & 0.21 & 3 & $\ldots$ & $\ldots$ & .. \\
\hline L4m04 & 23.58 & 0.53 & 5 & .. & $\ldots$ & .. & 22.14 & 0.00 & 1 \\
\hline $\mathrm{L} 4 \mathrm{n} 03$ & 23.72 & 0.11 & 4 & & & .. & 22.72 & 0.49 & 3 \\
\hline L4n04 & 23.60 & 0.17 & 4 & 22.45 & 0.11 & 4 & 22.50 & 0.00 & 1 \\
\hline L4n05 & 23.69 & 0.24 & 4 & $\ldots$ & $\ldots$ & .. & 23.05 & 0.15 & 2 \\
\hline L4n06 & 23.65 & 0.09 & 4 & $\ldots$ & $\ldots$ & .. & 23.49 & 0.79 & 3 \\
\hline L4o01 & 22.98 & 0.09 & 4 & 22.21 & 0.39 & 3 & 21.95 & 0.76 & 3 \\
\hline L4p01 & 23.90 & 0.13 & 3 & 23.48 & 0.41 & 3 & $\ldots$ & $\ldots$ & .. \\
\hline L4p02 & 23.83 & 0.20 & 4 & 23.07 & 0.14 & 2 & $\ldots$ & $\ldots$ & .. \\
\hline L4p03 & 23.17 & 0.11 & 4 & 22.45 & 0.14 & 4 & 22.09 & 0.11 & 2 \\
\hline L4p04PD & 21.96 & 0.14 & 4 & 22.33 & 1.43 & 4 & 21.58 & 0.09 & 3 \\
\hline L4p05 & 23.57 & 0.08 & 4 & 22.74 & 0.08 & 6 & 22.38 & 0.16 & 3 \\
\hline L4p06PD & 22.34 & 0.10 & 4 & 21.81 & 0.23 & 4 & 21.56 & 0.30 & 3 \\
\hline L4p07 & 22.32 & 0.34 & 4 & .. & & .. & 24.08 & 0.37 & 3 \\
\hline L4p08PD & 23.81 & 0.21 & 4 & $\cdots$ & & .. & 22.87 & 0.14 & 3 \\
\hline
\end{tabular}

Table 7

(Continued)

\begin{tabular}{|c|c|c|c|c|c|c|c|c|c|}
\hline Object & $g$ & $\sigma_{g}$ & $N_{g}$ & $r$ & $\sigma_{r}$ & $N_{r}$ & $i$ & $\sigma_{i}$ & \\
\hline L4p09 & 23.61 & 0.21 & 4 & 22.88 & 0.17 & 7 & 22.62 & 0.14 & 2 \\
\hline $\mathrm{L} 4 \mathrm{q} 03$ & .57 & 0.07 & 3 & 23.01 & 0.09 & 7 & 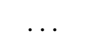 & 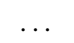 & \\
\hline $\mathrm{L} 4 \mathrm{q} 05$ & 23.56 & 0.11 & 4 & 23.04 & 0.10 & 3 & 22.84 & 0.41 & \\
\hline L4q06 & 23.97 & 0.24 & 4 & 23.54 & 0.19 & 3 & 23.83 & 0.43 & \\
\hline 4q09 & 4.11 & 0.12 & 4 & 23.11 & 0.01 & 3 & 22.72 & 0.23 & \\
\hline L4q10 & 3.54 & 0.27 & 4 & $\ldots$ & $\ldots$ & .. & 22.44 & 0.03 & \\
\hline $\mathrm{L} 4 \mathrm{q} 11$ & .92 & 0.14 & 4 & $\ldots$ & $\ldots$ &.. & 22.81 & 0.16 & \\
\hline $\mathrm{L} 4 \mathrm{q} 12 \mathrm{PI}$ & 24.11 & 0.09 & 3 & .. & $\ldots$ &.. & & & \\
\hline $\mathrm{L} 4 \mathrm{q} 14$ & 23.59 & 0.15 & 7 & $\ldots$ & $\ldots$ & .. & 22.89 & 0.34 & \\
\hline $\mathrm{L} 4 \mathrm{q} 15$ & 24.04 & 0.24 & 4 & $\ldots$ & $\ldots$ &.. & 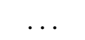 & & \\
\hline L4q16 & 23.71 & 0.17 & 4 & $\ldots$ & . &.. & 20.17 & 0.00 & \\
\hline $\mathrm{L} 4 \mathrm{v} 01$ & 24.18 & 0.19 & 4 & 24.00 & 1.07 & 2 & $\ldots$ & & \\
\hline $\mathrm{v} 02$ & 23.80 & 0.02 & 4 & 23.01 & 0.27 & 3 & $\ldots$ & $\ldots$ & \\
\hline 4v03 & 22.83 & 0.12 & 4 & 21.95 & 0.09 & 2 & & & \\
\hline 4v04 & 24.13 & 0.09 & 4 & 23.32 & 0.22 & 3 & 23.45 & 0.23 & \\
\hline 4v05 & 24.11 & 0.17 & 4 & 23.32 & 0.12 & 2 & 23.04 & 0.07 & \\
\hline v06 & 66 & 0.15 & 4 & 23.03 & 0.11 & 3 & 22.51 & 0.12 & \\
\hline L4v08 & 5 & 0.16 & 4 & 23.17 & 0.27 & 2 & 23.16 & & \\
\hline & & 0. & 5 & & & 4 & & & \\
\hline & & & 4 & & & 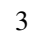 & & & \\
\hline $\mathrm{L} 4 \mathrm{v} 11$ & & & 4 & 2 & & 4 & 8 & & \\
\hline & & & 4 & 2 & & & 0 & & \\
\hline & & & 4 & 2 & & & 22.32 & 0.02 & \\
\hline & & & 4 & 7 & & 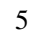 & & & \\
\hline 18 & & 2 & 3 & 22.66 & 0.15 & 4 & 22.57 & 0.27 & \\
\hline 03 & 6 & & 4 & $\ldots$ & $\ldots$ &.. & $\ldots$ & $\ldots$ & \\
\hline & & & 3 & & $\ldots$ &.. & 23.48 & 0.21 & \\
\hline & & & 4 & . . & $\ldots$ &.. & $\ldots$ & $\ldots$ & \\
\hline & & & 4 & & &.. & .. & & \\
\hline & 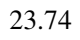 & 0 . & 4 & & &.. & 23.20 & 0.14 & \\
\hline & & & 4 & & &.. & 23.96 & 0.32 & \\
\hline & & & 4 & & $\ldots$ &.. & . . & & \\
\hline 14 & & & 5 & 23.53 & 0.38 & 3 & $\ldots$ & & \\
\hline & & & 3 & $\ldots$ & $\ldots$ &.. & $\ldots$ & $\ldots$ & \\
\hline 2 & 2 & & 4 & $\ldots$ & $\ldots$ &.. & $\ldots$ & $\ldots$ & \\
\hline & 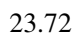 & & 5 & $\ldots$ & $\ldots$ &.. & $\ldots$ & $\ldots$ & \\
\hline 8 & ) & 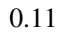 & 3 & $\ldots$ & $\ldots$ & .. & $\ldots$ & .. & \\
\hline & 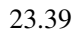 & & 4 & $\ldots$ & $\ldots$ & .. & . & .. & \\
\hline & ? & & 3 & $\cdots$ & $\cdots$ &.. & . & $\cdots$ & \\
\hline & & & 3 & & $\cdots$ & .. & $\ldots$ & & \\
\hline & & & 3 & & & .. & & $\ldots$ & \\
\hline 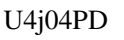 & & & 5 & 23.25 & 0.25 & 4 & 22.98 & 0.12 & \\
\hline & & & 2 & 23.29 & 0.17 & 6 & & & \\
\hline & & & 4 & .. & & .. & 0 & & \\
\hline 02 & 2 & 0.2 & 4 & $\ldots$ & .. & .. & 21.50 & 1.11 & \\
\hline & 2 & & 3 & $\ldots$ & $\ldots$ &.. & 23.13 & 0.13 & \\
\hline & 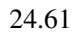 & & 3 & $\ldots$ & $\ldots$ & .. & $\ldots$ & $\ldots$ & \\
\hline 13 & 0 & L & 3 & $\ldots$ & $\ldots$ & .. & 22.85 & 0.19 & \\
\hline & & & 2 & 22.65 & 0.07 & 4 & 22.42 & 0.06 & \\
\hline & & & J & 23.27 & 0.05 & 3 & 23.01 & 0.15 & \\
\hline & & & 5 & $\ldots$ & $\ldots$ & .. & 22.68 & & \\
\hline 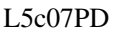 & & & 5 & 22.17 & 0.12 & 2 & 22.01 & 0. & \\
\hline & & & 6 & 22.66 & 0.20 & 4 & 22.67 & 0.07 & \\
\hline & & & 3 & & & .. & & & \\
\hline & & & 4 & 23.16 & 0.14 & 3 & & & \\
\hline & & & 4 & $\ldots$ & $\ldots$ & .. & 9 & & \\
\hline 130 & 23.80 & 0 & 4 & $\ldots$ & $\ldots$ & .. & 23.16 & 0.12 & \\
\hline $5 \mathrm{c} 14$ & 23.41 & 0.09 & 3 & $\ldots$ & $\ldots$ &.. & 22.61 & 0.07 & \\
\hline & 24.16 & 0.16 & 4 & 23.27 & 0.57 & 4 & 23.27 & 0.15 & \\
\hline & 23.08 & & 4 & 22.67 & 0.05 & 3 & 22.63 & 0.11 & \\
\hline & 23.84 & & 3 & & & .. & & .. & \\
\hline $10 \mathrm{~L}$ & 2 & & 3 & 23.24 & 0.28 & 4 & 23.17 & 0.08 & \\
\hline & & & 4 & 23.69 & 0.59 & 4 & 22.52 & 0.97 & \\
\hline & 23. & 0.20 & 3 & 22.84 & 0.10 & 4 & 22.79 & 0.14 & \\
\hline 5 & & 0.19 & 4 & 22.83 & 0.07 & 3 & 22.48 & 0.03 & \\
\hline L5c23 & 24.19 & 0.09 & 3 & 23.37 & 0.07 & 4 & 23.27 & 0.16 & \\
\hline
\end{tabular}


Table 7

(Continued)

\begin{tabular}{|c|c|c|c|c|c|c|c|c|c|}
\hline Object & $g$ & $\sigma_{g}$ & $N_{g}$ & $r$ & $\sigma_{r}$ & $N_{r}$ & $i$ & $\sigma_{i}$ & $N_{i}$ \\
\hline L5c24PD & 23.84 & 0.40 & 3 & $\ldots$ & . & .. & 22.86 & 0.90 & 4 \\
\hline L5i01 & 23.66 & 0.27 & 4 & $\ldots$ & $\ldots$ &.. & $\ldots$ & $\ldots$ & .. \\
\hline L5i02PD & 23.85 & 0.16 & 4 & $\ldots$ & $\ldots$ &.. & $\ldots$ & $\ldots$ & .. \\
\hline L5i03PD & 23.77 & 0.21 & 4 & 22.97 & 0.58 & 3 & $\ldots$ & $\ldots$ & .. \\
\hline L5i04 & 23.07 & 0.21 & 7 & $\ldots$ & $\ldots$ &.. & $\ldots$ & $\ldots$ & .. \\
\hline L5i05 & 23.81 & 0.21 & 4 & $\ldots$ & $\ldots$ &.. & $\ldots$ & $\ldots$ & .. \\
\hline L5i06PD & 23.12 & 0.05 & 4 & $\ldots$ & $\ldots$ &.. & 22.41 & 0.20 & 3 \\
\hline L5i08 & 23.20 & 0.61 & 4 & $\ldots$ & $\ldots$ &.. & 22.41 & 0.08 & 3 \\
\hline L5j02 & 23.29 & 0.11 & 5 & 22.20 & 0.18 & 3 & $\ldots$ & $\ldots$ & .. \\
\hline L5j03 & 23.16 & 0.07 & 3 & 22.50 & 0.10 & 3 & 22.23 & 0.05 & 4 \\
\hline L5j04 & 22.51 & 0.11 & 5 & 21.64 & 0.18 & 3 & 21.52 & 0.15 & 2 \\
\hline L5r01 & 23.65 & 0.22 & 4 & $\ldots$ & $\ldots$ &.. & $\ldots$ & $\ldots$ & .. \\
\hline L5s01PD & 20.84 & 0.02 & 3 & $\ldots$ & $\ldots$ &.. & $\ldots$ & $\ldots$ & .. \\
\hline $15 \mathrm{c} 01$ & 24.05 & 0.55 & 5 & $\ldots$ & $\ldots$ &.. & $\ldots$ & $\ldots$ & .. \\
\hline $15 \mathrm{c} 04$ & 24.21 & 0.33 & 6 & $\ldots$ & $\ldots$ &.. & $\ldots$ & $\ldots$ & .. \\
\hline U5c17PD & 24.18 & 0.30 & 3 & $\ldots$ & $\ldots$ &.. & 23.46 & 0.14 & 3 \\
\hline U5j01PD & 23.83 & 0.16 & 5 & 23.37 & 0.24 & 3 & $\ldots$ & $\ldots$ & .. \\
\hline U5j06 & 23.67 & 0.23 & 5 & 23.11 & 0.25 & 4 & 23.06 & 0.16 & 3 \\
\hline u $5 \mathrm{c} 09$ & 24.35 & 0.21 & 2 & $\ldots$ & $\ldots$ &.. & $\ldots$ & $\ldots$ & .. \\
\hline u5i07 & 24.29 & 0.41 & 4 & $\ldots$ & $\ldots$ &.. & 22.92 & 0.21 & 3 \\
\hline u5j05 & 23.50 & 0.06 & 5 & & &.. & 22.83 & 0.09 & 2 \\
\hline $\mathrm{L} 7 \mathrm{a} 02$ & 23.50 & 0.33 & 3 & $\ldots$ & $\ldots$ &.. & 22.74 & 0.13 & 4 \\
\hline $\mathrm{L} 7 \mathrm{a} 03$ & 23.80 & 0.07 & 4 & 23.26 & 0.12 & 4 & 23.27 & 0.15 & 3 \\
\hline L7a04PD & 23.29 & 0.11 & 4 & $\ldots$ & $\ldots$ &.. & 21.92 & 0.09 & 3 \\
\hline $\mathrm{L} 7 \mathrm{a} 05$ & 23.68 & 0.20 & 4 & 22.85 & 0.00 & 1 & 22.82 & 0.38 & 3 \\
\hline $\mathrm{L} 7 \mathrm{a} 06$ & 23.78 & 0.13 & 4 & $\ldots$ & $\ldots$ &.. & 22.92 & 0.12 & 4 \\
\hline $\mathrm{L} 7 \mathrm{a} 07$ & 23.43 & 0.20 & 4 & 22.75 & 0.11 & 3 & 22.55 & 0.11 & 4 \\
\hline L7a10 & 23.63 & 0.04 & 4 & 23.35 & 0.22 & 4 & 23.26 & 0.11 & 3 \\
\hline L7a11PD & 23.34 & 0.16 & 5 & 22.64 & 0.10 & 4 & 22.67 & 0.10 & 4 \\
\hline $17 \mathrm{a} 12$ & 23.86 & 0.13 & 6 & $\ldots$ & $\ldots$ &.. & $\ldots$ & $\ldots$ & .. \\
\hline $\mathrm{U} 7 \mathrm{a} 01$ & 24.13 & 0.16 & 4 & 23.29 & 0.26 & 4 & 22.94 & 0.27 & 2 \\
\hline $\mathrm{U} 7 \mathrm{a} 08$ & 24.00 & 0.18 & 4 & 23.62 & 0.14 & 4 & 23.25 & 0.32 & 3 \\
\hline $\mathrm{U} 7 \mathrm{a} 09$ & 24.11 & 0.21 & 3 & 23.45 & 0.16 & 4 & 23.41 & 0.28 & 3 \\
\hline
\end{tabular}

Notes. "L" objects are the tracked, characterized (i.e., with flux above the $40 \%$ detection-efficiency level) objects of CFEPS. "l" objects are the non-tracked, characterized objects of CFEPS. There is no ephemeris-based bias in those losses. Most of them were not recovered at checkup (either too faint or sheared out of field coverage westward). "U" objects are the tracked, non-characterized (i.e., with flux below $40 \%$ detection-efficiency level) objects of CFEPS. "u" objects are the non-tracked, non-characterized objects of CFEPS. Magnitudes listed for photometric observations from CFHT. Some numbers are missing because the corresponding object was not re-observed in a particular filter from CFHT in photometric conditions. This is the case for lost objects, some of the PD objects which we did not try to track, or objects tracked solely at other facilities. $g, r, i$ columns give the apparent magnitude of the object in the correspondent filter. $\sigma_{x}$ is the uncertainty on the magnitude in filter $x . N_{x}$ is the number of measurements in filter $x$ used to derive the apparent magnitude and its uncertainty.

debiased objects fainter than 24 would push the population estimate down. For the main-belt model used by Trujillo et al., $m_{R}=24$ corresponds to $H_{R} \sim 7.7$ or $H_{g} \sim 8.5$. Hence their population estimate is probably more applicable to that limit but not to smaller sizes. With $q=4$ or $\alpha=0.6$, the population estimates of Trujillo et al. (2001) is

$$
N_{\text {Trujillo }}\left(H_{g} \leqslant 8.5\right)=8300_{-600}^{+1200} \cdot[3 \sigma]
$$

Restricting our model to the same phase space and extrapolating our population estimate out to $H_{g} \leqslant 8.5$, we obtain

$$
N_{\text {All }}\left(H_{g} \leqslant 8.5\right)=19,000_{-3700}^{+4100},[95 \% \text { confidence }]
$$

a factor of two larger than Trujillo et al. (2001). However, this last number is an extrapolation beyond the limit to which CFEPS really measured the population. A more secure comparison from our point of view is done for $H_{g} \leqslant 8.0$. The numbers become

$$
N_{\text {Trujillo }}\left(H_{g} \leqslant 8.0\right)=4200_{-300}^{+600} \text {, }
$$

and

$$
N_{\text {All }}\left(H_{g} \leqslant 8.0\right)=5500_{-1100}^{+1300} \cdot[95 \% \text { confidence }] .
$$

Hence we marginally agree with Trujillo et al. (2001) at $H_{g} \leqslant 8.0$. At $H_{g}>8.0$, an extrapolation of our result (using our two $H$ slopes determined at larger sizes) rapidly diverges from the Trujillo et al. (2001) estimate; if the slope does indeed drop near $H \sim 8$ (or $R \simeq 24$ ) to a shallower slope (Fuentes \& Holman 2008) then the two estimates are less discrepant.

\section{REFERENCES}

Allen, R. L., Bernstein, G. M., \& Malhotra, R. 2001, ApJ, 549, L241

Allen, R. L., Bernstein, G. M., \& Malhotra, R. 2002, AJ, 124, 2949

Allen, R. L., Gladman, B., Kavelaars, J. J., et al. 2006, ApJ, 640, L83 Bernstein, G. M., Trilling, D. E., Allen, R. L., et al. 2004, AJ, 128, 1364

Bowell, E., Hapke, B., Domingue, D., et al. 1989, in Asteroids II, ed. R. P. Binzel, T. Gehrels, \& M. S. Matthews (Tucson, AZ: Univ. Arizona Press), 524

Brown, M. E. 2001, AJ, 121, 2804

Brown, M. E. 2008, in The Solar System Beyond Neptune, ed. M. A. Barucci, H. Boehnhardt, D. P. Cruikshank, \& A. Morbidelli (LPI; Tuscon, AZ: Univ. Arizona Press), 335

Brown, M. E., Barkume, K. M., Ragozzine, D., \& Schaller, E. L. 2007, Nature, 446, 294

Brown, M. E., \& Pan, M. 2004, AJ, 127, 2418

Chiang, E. I. 2002, ApJ, 573, L65

Chiang, E. I., Jordan, A. B., Millis, R. L., et al. 2003a, AJ, 126, 430

Chiang, E. I., Lovering, J. R., Millis, R. L., et al. 2003b, Earth Moon Planets, 92, 49

Duncan, M. J., \& Levison, H. F. 1997, Science, 276, 1670

Duncan, M. J., Levison, H. F., \& Budd, S. M. 1995, AJ, 110, 3073

Elliot, J. L., Kern, S. D., Clancy, K. B., et al. 2005, AJ, 129, 1117

Fraser, W. C., Brown, M. E., \& Schwamb, M. E. 2010, Icarus, 210, 944

Fraser, W. C., \& Kavelaars, J. J. 2009, AJ, 137, 72

Fuentes, C. I., George, M. R., \& Holman, M. J. 2009, ApJ, 696, 91

Fuentes, C. I., \& Holman, M. J. 2008, AJ, 136, 83

Fukugita, M., Ichikawa, T., Gunn, J. E., et al. 1996, AJ, 111, 1748

Gladman, B., \& Chan, C. 2006, ApJ, 643, L135

Gladman, B., Holman, M., Grav, T., et al. 2002, Icarus, 157, 269

Gladman, B., Kavelaars, J., Allen, L., et al. 2006, Minor Planet Electronic Circulars, 2006-H29

Gladman, B., Kavelaars, J., Petit, J.-M., et al. 2009, ApJ, 697, L91

Gladman, B., Kavelaars, J. J., Petit, J.-M., et al. 2001, AJ, 122, 1051

Gladman, B., et al. 2011, AJ, submitted

Gladman, B. J., Marsden, B. G., \& van Laerhoven, C. 2008, in The Solar System Beyond Neptune, ed. A. Barucci, H. Boehnhardt, D. Cruikshank, \& A. Morbidelli (LPI; Tucson, AZ: Univ. Arizona Press), 43

Gomes, R. S. 2003, Icarus, 161, 404

Gomes, R. S., Morbidelli, A., \& Levison, H. F. 2004, Icarus, 170, 492

Gulbis, A. A. S., Elliot, J. L., Adams, E. R., et al. 2010, AJ, 140, 350

Gulbis, A. A. S., Elliot, J. L., \& Kane, J. F. 2006, Icarus, 183, 168

Hahn, J. M., \& Malhotra, R. 2005, AJ, 130, 2392

Hainaut, O. R., \& Delsanti, A. C. 2002, A\&A, 389, 641

Ida, S., Larwood, J., \& Burkert, A. 2000, ApJ, 528, 351

Information Technology Laboratory, N. 2011, NIST/SEMATECH e-Handbook of Statistical Methods, Technical Report, National Institute of Standards and Technology, U.S. Commerce Department

Ivezic, Z., Axelrod, T., Brandt, W. N., et al. 2008, Serb. Astron. J., 176, 1

Jewitt, D., Luu, J., \& Chen, J. 1996, AJ, 112, 1225

Jewitt, D., Luu, J., \& Trujillo, C. 1998, AJ, 115, 2125

Jewitt, D. C., \& Luu, J. X. 1995, AJ, 109, 1867

Johnstone, D., Hollenbach, D., \& Bally, J. 1998, ApJ, 499, 758

Jones, R. L., Gladman, B., Petit, J.-M., et al. 2006, Icarus, 185, 508

Jones, R. L., Parker, J. W., Bieryla, A., et al. 2010, AJ, 139, 2249

Kavelaars, J., Allen, L., Gladman, B., et al. 2006a, Minor Planet Electronic Circulars, 2006-H35

Kavelaars, J., Allen, L., Gladman, B., et al. 2011, Minor Planet Electronic Circulars, 2011 
Kavelaars, J., Jones, L., Gladman, B., Parker, J. W., \& Petit, J.-M. 2008, in The Solar System Beyond Neptune, ed. M. A. Barucci, H. Boehnhardt, D. P. Cruikshank, \& A. Morbidelli (Tucson, AZ: Univ. Arizona Press), 59

Kavelaars, J., et al. 2006b, Minor Planet Electronic Circulars, 2006-H30

Kavelaars, J. J., Jones, R. L., Gladman, B. J., et al. 2009, AJ, 137, 4917

Larsen, J. A., Gleason, A. E., Danzl, N. M., et al. 2001, AJ, 121, 562

Leinhardt, Z. M., Marcus, R. A., \& Stewart, S. T. 2010, ApJ, 714, 1789

Levison, H. F., Morbidelli, A., Vanlaerhoven, C., Gomes, R., \& Tsiganis, K. 2008, Icarus, 196, 258

Lykawka, P. S., \& Mukai, T. 2007, Icarus, 189, 213

Magnier, E. A., \& Cuillandre, J.-C. 2004, PASP, 116, 449

Malhotra, R. 1993, Nature, 365, 819

Mann, R. K., \& Williams, J. P. 2009, ApJ, 694, L36

Mann, R. K., \& Williams, J. P. 2010, ApJ, 725, 430

Millis, R. L., Buie, M. W., Wasserman, L. H., et al. 2002, AJ, 123, 2083

Morbidelli, A., Bottke, W., Nesvorný, D., \& Levison, H. 2009a, Icarus, 204 558

Morbidelli, A., Brasser, R., Tsiganis, K., Gomes, R., \& Levison, H. F. 2009b, A\&A, 507, 1041

Morbidelli, A., Emel'yanenko, V. V., \& Levison, H. F. 2004, MNRAS, 355, 935

Morbidelli, A., \& Levison, H. F. 2004, AJ, 128, 2564

Morbidelli, A., Levison, H. F., Tsiganis, K., \& Gomes, R. 2005, Nature, 435, 462
Nagasawa, M., \& Ida, S. 2000, AJ, 120, 3311

Parker, A. H., \& Kavelaars, J. J. 2010, ApJ, 722, L204

Petit, J.-M., Holman, M., Scholl, H., Kavelaars, J., \& Gladman, B. 2004, MNRAS, 347, 471

Petit, J.-M., Holman, M. J., Gladman, B. J., et al. 2006, MNRAS, 365, 429

Petit, J.-M., Kavelaars, J. J., Gladman, B., \& Loredo, T. 2008, in The Solar System Beyond Neptune, ed. M. A. Barucci, H. Boehnhardt, D. P. Cruikshank, \& A. Morbidelli (Tucson, AZ: Univ. Arizona Press), 71

Romanishin, W., Tegler, S. C., \& Consolmagno, G. J. 2010, AJ, 140, 29

Schwamb, M. E., Brown, M. E., Rabinowitz, D. L., \& Ragozzine, D. 2010, ApJ, 720,1691

Stark, C. C., \& Kuchner, M. J. 2010, BAAS, 42, 527

Stepinski, T. F., \& Valageas, P. 1996, A\&A, 309, 301

Stepinski, T. F., \& Valageas, P. 1997, A\&A, 319, 1007

Thommes, E. W., Duncan, M. J., \& Levison, H. F. 1999, Nature, 402, 635

Throop, H. B., \& Bally, J. 2005, ApJ, 623, L149

Trujillo, C. A., \& Brown, M. E. 2001, ApJ, 554, L95

Trujillo, C. A., \& Brown, M. E. 2003, Earth Moon Planets, 92, 99

Trujillo, C. A., Jewitt, D. C., \& Luu, J. X. 2000, ApJ, 529, L103

Trujillo, C. A., Jewitt, D. C., \& Luu, J. X. 2001, AJ, 122, 457

Tsiganis, K., Gomes, R., Morbidelli, A., \& Levison, H. F. 2005, Nature, 435 459

Youdin, A. N. 2011, ApJ, 731, 99 